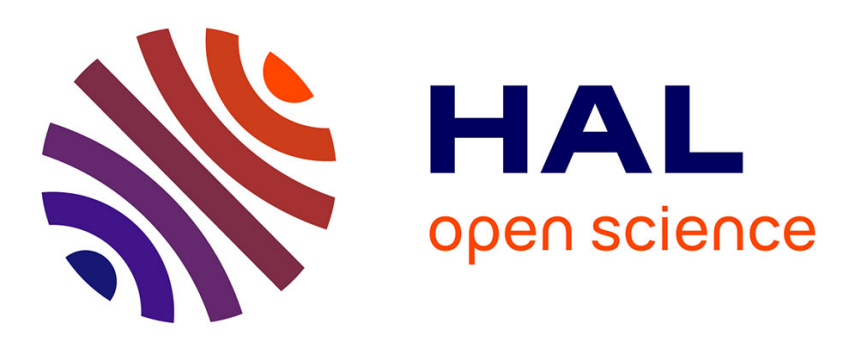

\title{
A high-order interpolation for the finite volume method: The Coupled Least Squares reconstruction
}

Florian Haider, Bernard Courbet, Jean-Pierre Croisille

\section{To cite this version:}

Florian Haider, Bernard Courbet, Jean-Pierre Croisille. A high-order interpolation for the finite volume method: The Coupled Least Squares reconstruction. Computers and Fluids, 2018, 176, pp.2039. 10.1016/j.compfluid.2018.09.009 . hal-01879576

\section{HAL Id: hal-01879576 https://hal.science/hal-01879576}

Submitted on 24 Sep 2018

HAL is a multi-disciplinary open access archive for the deposit and dissemination of scientific research documents, whether they are published or not. The documents may come from teaching and research institutions in France or abroad, or from public or private research centers.
L'archive ouverte pluridisciplinaire HAL, est destinée au dépôt et à la diffusion de documents scientifiques de niveau recherche, publiés ou non, émanant des établissements d'enseignement et de recherche français ou étrangers, des laboratoires publics ou privés. 


\title{
A High-Order interpolation for the Finite Volume Method: the Coupled Least Squares Reconstruction
}

\author{
Florian Haider*, Bernard Courbet* and Jean-Pierre Croisille** \\ * Onera, 92320 Châtillon, France, \\ bernard.courbet@onera.fr,florian.haider@onera.fr. \\ ** IECL, UMR CNRS 7502, Mathématiques, Univ. de Lorraine, France, jean-pierre.croisille@univ-lorraine.fr
}

\begin{abstract}
We consider the Finite Volume Method on irregular grids for conservation laws with a particular polynomial reconstruction. This reconstruction is based on a least squares approach to compute consistent approximations of the first, second and third order derivatives. The resulting reconstruction is cubic. It is called the Coupled Least Squares reconstruction. It is obtained by a three stages iteration. At each iteration, only data located in a compact stencil, and not beyond, are accessed. A linear stability analysis is given in the case of regular and irregular one-dimensional grids. Numerical results for various problems, including shock tubes, vortex and accoustic wave propagation, support the interest of this approach. The reconstruction algorithm is presented in detail in the one dimensional case. An outline of the multidimensional case in also given.
\end{abstract}

This work was announced in [14].

Keywords: Finite volume method - High order method - k-exact method - Cubic reconstruction Numerical flux - Linear stability - Irregular grid - Coupled Least Squares.

\section{Introduction}

\subsection{Finite volume schemes with high order reconstruction}

In $[13,14]$, a high order reconstruction approach for finite volume approximations was introduced for the purpose of gas dynamics simulations on general polyhedral grids. This approach, which is called the Coupled Least Squares reconstruction, belongs to the $k$ - exact methods [4]. The purpose of this paper is to give a detailed account of a particular algorithm of the reconstruction. The presentation is mainly restricted to the one dimensional context to emphasize the logic of the numerical procedure.

Consider the conservation law

$$
\partial_{t} u+\partial_{x} f(u)=0
$$

We start from the discrete in space, continuous in time, integral version of (1.1):

$$
\frac{d v_{\alpha}(t)}{d t}=-\frac{1}{\left|\mathcal{T}_{\alpha}\right|}\left[f_{\alpha+1 / 2}(t)-f_{\alpha-1 / 2}(t)\right]
$$

The grid is made of cells $\mathcal{T}_{\alpha}$ and is depicted on Fig 1.1. In (1.2) $t \mapsto v_{\alpha}(t)$ approximates $t \mapsto \bar{u}_{\alpha}(t)$ defined by

$$
\bar{u}_{\alpha}(t)=\frac{1}{\left|\mathcal{T}_{\alpha}\right|} \int_{\mathcal{T}_{\alpha}} u(x, t) d x
$$




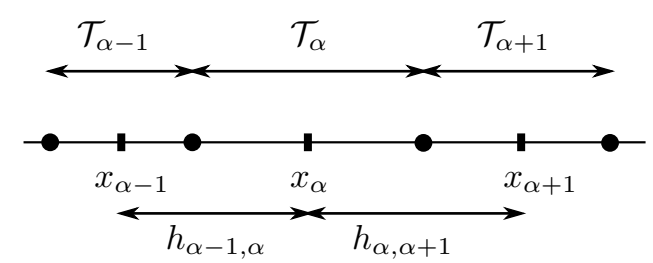

Figure 1.1: The cell $\mathcal{T}_{\alpha}$ and the two neighboor cells $\mathcal{T}_{\alpha \pm 1}$. The barycenters of the cells $\mathcal{T}_{\alpha \pm 1}$ and $\mathcal{T}_{\alpha}$ are the points $x_{\alpha \pm 1}$ and $x_{\alpha}$, repectively. In addition, $h_{\alpha-1, \alpha}=x_{\alpha}-x_{\alpha-1}$ and $h_{\alpha, \alpha+1}=x_{\alpha+1}-x_{\alpha}$.

where $u(x, t)$ is the solution of (1.1). Throughout the paper, the numerical flux $t \mapsto f_{\alpha+1 / 2}(t)$ is of the form:

$$
f_{\alpha+1 / 2}(t)=F\left(w_{\alpha}[V(t)]\left(x_{\alpha+1 / 2}^{-}\right), w_{\alpha+1}[V(t)]\left(x_{\alpha+1 / 2}^{+}\right)\right) .
$$

where:

- The function $\left(u_{L}, u_{R}\right) \mapsto F\left(u_{L}, u_{R}\right)$ denotes some numerical flux function, typically the HLLC flux in the case of the Euler equations [29].

- The reconstruction $x \mapsto w_{\alpha}[V(t)](x)$ is an interpolant based on the vector $V(t)=\left[v_{1}(t), v_{2}(t), \ldots, v_{N}(t)\right]^{T}$.

The reconstruction expounded in this paper has the form of a Taylor expansion of $u(x)$ around the barycenter $x_{\alpha}$ of the cell $\mathcal{T}_{\alpha}$, i.e. (we take out the time dependency for clarity):

$$
w_{\alpha}[\overline{\mathfrak{u}}](x) \simeq u\left(x_{\alpha}\right)+u^{\prime}\left(x_{\alpha}\right)\left(x-x_{\alpha}\right)+\frac{1}{2} u^{\prime \prime}\left(x_{\alpha}\right)\left(x-x_{\alpha}\right)^{2}+\frac{1}{6} u^{\prime \prime \prime}\left(x_{\alpha}\right)\left(x-x_{\alpha}\right)^{3} .
$$

where $\overline{\mathfrak{u}}=\left[\bar{u}_{\alpha}\right]$ is the vector of averages of $u(x)$ over the cells $\mathcal{T}_{\alpha}$. The relation (1.5) is a 3 - exact reconstruction. The main task is to define a consistent approximation to $u^{\prime}\left(x_{\alpha}\right), u^{\prime \prime}\left(x_{\alpha}\right)$ and $u^{\prime \prime \prime}\left(x_{\alpha}\right)$, where $u(x)$ is supposed to be known by $\overline{\mathfrak{u}}$ only.

The main observation is the following: to preserve fourth order accuracy in (1.5), it is sufficient to calculate approximations of $u^{\prime}\left(x_{\alpha}\right), u^{\prime \prime}\left(x_{\alpha}\right)$ and $u^{\prime \prime \prime}\left(x_{\alpha}\right)$ with order 3,2 and 1 , respectively. It is the purpose of this paper to show how this can be obtained in several steps of an iterative procedure along which the accuracy is progessively enhanced. Moreover, at each step, there is an access only to data located in a small neighborhood of any given cell, and therefore it is called a compact k-exact reconstruction.

This paper is the companion paper of [14] where the multidimensional aspect on general polyhedral grids of our algorithm is addressed. This study has been initiated in [12], in the context of the package CEDRE, a parallel multisolver CFD code for aerothermochemistry ${ }^{1}$.

Our point of view is thus the one of a generic computational procedure for any finite volume code and for general compressible flows. For an overview on the $k$ - exact approach for high order computations in $\mathrm{CFD}$, compared to other approaches, we refer to the review paper [30].

\subsection{High order finite volume schemes}

We consider the finite volume method (1.2) for a conservation law (1.1) on a general irregular grid made of cells $\mathcal{T}_{\alpha}$, which support approximations $v_{\alpha}(t)$ of the averages $\bar{u}_{\alpha}(t)$. As explained in Section 1.1, we want to define the reconstructed polynomial $w_{\alpha}[V(t)](x)$ which is used in the numerical flux $f_{\alpha+1 / 2}(t)$ in (1.4). This polynomial must have the following properties (we drop the $t$ dependency).

\footnotetext{
${ }^{1}$ CEDRE is being developed at Onera, France, http://cedre.onera.fr
} 
First $w_{\alpha}[V](x)$ depends linearly on the data $v_{\beta}$ in the cells $\mathcal{T}_{\beta}$ belonging to a small neighborhood of $\mathcal{T}_{\alpha}$. In particular, it does not use gradient data as for example in the Discontinuous Galerkin approximation, but only finite volume data. Furthermore, no flow based local parameters are used in a nonlinear way to define $w_{\alpha}[V](x)$. Consequently, our reconstruction is decoupled from all nonlinear treatments such as slope limiting or monotonicity preserving procedures. Thus the reconstruction step is decoupled from the limitation procedure. The reconstruction step can be applied in principle to any finite volume code including complex fluid modelling such as turbulent, multiphasic, reactive, spherical flows, etc. With this approach, the nonlinear treatments are performed in a second step, after the reconstruction step. Having in view general CFD codes, the design of the reconstruction must of course be multidimensional. Moreover, it must allow an easy parallel implementation. This is obtained by mean of the procedure in several stages presented in Section 2 and Section 3.

\subsection{Related works}

Reconstruction beyond linear in the finite volume method on irregular grids has been a longstanding issue of great importance, both practical and theoretical in CFD. It takes place in the challenge to go beyond second order with the finite volume method. The problem was introduced in $[17,1]$ where various polynomial interpolations on triangles were studied. Quadratic reconstruction is undertaken in $[4,7]$. The series of studies $[22,21]$ explores the finite volume method with quadratic reconstruction for compressible Fluid Dynamics. In many works, the reconstruction is analyzed in conjunction with the limiter and with the positivity preserving property [3, 2], which are considered as constraints on the reconstruction. This is for example the case in the well known ENO/WENO procedure. In this approach, local high order reconstruction functions are based on stencils which vary according to local sensors related the solution, e.g. the total variation. We refer to [16, 18, 27, 28]. Another option [9] is where the reconstruction in finite volumes is considered in the general framework of the Discontinuous Galerkin approach. We also refer to the studies [19,31], with interest into application oriented simulations, where reconstructions beyond linear are presented. Finally we mention the recent study [23] where a variant of compact $k$-exact reconstruction is considered. In this study the authors use an upwinded scheme only up to third order and switch to a fourth order centered scheme in vorticity dominated regions. This is in contrast to our scheme which is fully upwinded up to fourth order independently of the flow regime.

\subsection{Outline}

The outline of the paper is as follows. In Section 2 we present the principle of our reconstruction algorithm. In Section 2.1, the general $d$ - dimensional framework is set up for the reconstructed cubic polynomial $w_{\alpha}[\overline{\mathfrak{u}}](\mathbf{x})$. From Section 2.2 on, we switch to the one dimensional case to present how the reconstruction uses at each step only data in the direct neighborhood of $\mathcal{T}_{\alpha}$. In Section 3 and Section 4 the detail of the algorithm to calculate the cubic reconstruction is summarized (algorithm 1). This is

based on fully centered approximate derivatives $\tilde{\sigma}_{\alpha}, \tilde{\theta}_{\alpha}$ and $\tilde{\psi}_{\alpha}$ in the cubic reconstruction (1.5) giving rise to a linearly stable semidiscrete system (1.2), even on irregular grids. Our analysis is based on the numerical computation of discrete spectra. Finally we show in Section 5 numerical results in one and multidimension illustrating our approach.

\section{Coupled Least Squares Reconstruction}

\subsection{The $d$ - dimensional case}

The Coupled Least Squares method is a particular method to implement a $k$ - exact reconstruction. This method has been already been introduced in [14]. Here we specifically focus on the piecewise cubic reconstruction (i.e $k=3$ ). Let $\Omega \subset \mathbb{R}^{d}, d \geq 1$ be a domain with periodic boundary conditions. We consider a grid, a priori irregular, consisting of $N$ general polyhedral cells $\mathcal{T}_{\alpha}$ as on Fig. 2.1. 


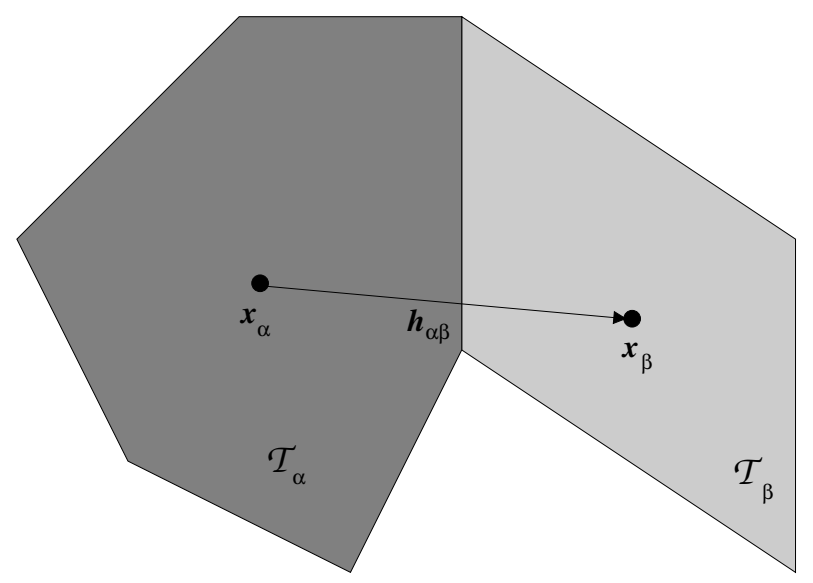

Figure 2.1: Two cells of a general polyhedral grid in the two dimensional case, $(d=2$

$$
\Omega=\bigcup_{\alpha=1}^{N} \mathcal{T}_{\alpha} .
$$

The tensor notation used in this section is summarized in Appendix 8. The reconstruction algorithm proceeds in several steps. At each step, the only data that are used are located in a small neighborhood $\mathbb{W}_{\alpha}$ of the cell $\mathcal{T}_{\alpha}$. The set $\mathbb{W}_{\alpha}$ is a parameter of the method. A typical choice of $\mathbb{W}_{\alpha}$ is the first neighborhood ${ }^{2}$ defined by

$$
\mathbb{V}_{\alpha}^{(1)}=\left\{\beta \in[1: N], \mid \mathcal{T}_{\beta} \text { is adjacent to } \mathcal{T}_{\alpha}\right\}
$$

Let $\mathbf{x} \in \Omega \mapsto u(\mathbf{x})$ be a given regular function. The value of $u$ and of the first, second and third order derivatives at $\mathbf{x}_{\alpha}$ are denoted by

$$
u_{\alpha}=u\left(\mathbf{x}_{\alpha}\right), \quad D^{(1)} u_{\alpha}=D_{\mathbf{x}}^{(1)} u\left(\mathbf{x}_{\alpha}\right), \quad D^{(2)} u_{\alpha}=D_{\mathbf{x}}^{(2)} u\left(\mathbf{x}_{\alpha}\right), \quad D^{(3)} u_{\alpha}=D_{\mathbf{x}}^{(3)} u\left(\mathbf{x}_{\alpha}\right) .
$$

The derivative $D^{(l)} u_{\alpha}$ is a symmetric tensor of order $l$. We also note the mean value

$$
\bar{u}_{\alpha}=\frac{1}{\left|\mathcal{T}_{\alpha}\right|} \int_{\mathcal{T}_{\alpha}} u(\mathbf{x}) d \mathbf{x}
$$

Consider the particular case where $u(\mathbf{x})=p(\mathbf{x})$ is a cubic polynomial. The values $\bar{p}_{\beta}-\bar{p}_{\alpha}$ and the three derivatives $D^{(l)} p_{\alpha}, l=1,2,3$, satisfy for all $\beta \in \mathbb{W}_{\alpha}$ the following Taylor expansions (see Appendix 8 for the notation),

$$
\left\{\begin{array}{l}
\bar{p}_{\beta}-\bar{p}_{\alpha}=D^{(1)} p_{\alpha} \bullet\left(\mathbf{z}_{\alpha \beta}^{(1)}-\mathbf{x}_{\alpha}^{(1)}\right)+\frac{1}{2} D^{(2)} p_{\alpha} \bullet\left(\mathbf{z}_{\alpha \beta}^{(2)}-\mathbf{x}_{\alpha}^{(2)}\right)+\frac{1}{6} D^{(3)} p_{\alpha} \bullet\left(\mathbf{z}_{\alpha \beta}^{(3)}-\mathbf{x}_{\alpha}^{(3)}\right) \\
D^{(1)} p_{\beta}=D^{(1)} p_{\alpha}+D^{(2)} p_{\alpha} \bullet \mathbf{h}_{\alpha \beta}^{(1)}+\frac{1}{2} D^{(3)} p_{\alpha} \bullet \mathbf{h}_{\alpha \beta}^{(2)} \\
D^{(2)} p_{\beta}=D^{(2)} p_{\alpha}+D^{(3)} p_{\alpha} \bullet \mathbf{h}_{\alpha \beta}^{(1)}
\end{array}\right.
$$

\footnotetext{
${ }^{2}$ The first neighborhood of the cell $\mathcal{T}_{\alpha}$ is also called the direct or the Von Neumann neighborhood.
} 
For a general function $u(\mathbf{x})$ these Talor expansions become

$$
\left\{\begin{array}{l}
\bar{u}_{\beta}-\bar{u}_{\alpha}=D^{(1)} u_{\alpha} \bullet\left(\mathbf{z}_{\alpha \beta}^{(1)}-\mathbf{x}_{\alpha}^{(1)}\right)+\frac{1}{2} D^{(2)} u_{\alpha} \bullet\left(\mathbf{z}_{\alpha \beta}^{(2)}-\mathbf{x}_{\alpha}^{(2)}\right)+\frac{1}{6} D^{(3)} u_{\alpha} \bullet\left(\mathbf{z}_{\alpha \beta}^{(3)}-\mathbf{x}_{\alpha}^{(3)}\right)+O\left(h^{4}\right), \\
D^{(1)} u_{\beta}=D^{(1)} u_{\alpha}+D^{(2)} u_{\alpha} \bullet \mathbf{h}_{\alpha \beta}^{(1)}+\frac{1}{2} D^{(3)} u_{\alpha} \bullet \mathbf{h}_{\alpha \beta}^{(2)}+O\left(h^{3}\right) \\
D^{(2)} u_{\beta}=D^{(2)} u_{\alpha}+D^{(3)} u_{\alpha} \bullet \mathbf{h}_{\alpha \beta}^{(1)}+O\left(h^{2}\right) .
\end{array}\right.
$$

Therefore, due to the $O\left(h^{p}\right)$ terms in (2.6), the quantities $\bar{u}_{\beta}-\bar{u}_{\alpha}, D^{(1)} u_{\alpha}, D^{(2)} u_{\alpha}$ and $D^{(3)} u_{\alpha}$ are approximate solutions of the system (2.5). Consider now the problem to approximate the three derivatives $D^{(1)} u_{\alpha}, D^{(2)} u_{\alpha}$ and $D^{(3)} u_{\alpha}$ by consistent values. We look for symmetric tensors called $\tilde{\boldsymbol{\sigma}}_{\alpha}, \tilde{\boldsymbol{\theta}}_{\alpha}$ and $\tilde{\boldsymbol{\psi}}_{\alpha}$ satisfying

$$
\left\{\begin{array}{c}
\tilde{\boldsymbol{\sigma}}_{\alpha}=D^{(1)} u_{\alpha}+O\left(h^{3}\right) \\
\tilde{\boldsymbol{\theta}}_{\alpha}=D^{(2)} u_{\alpha}+O\left(h^{2}\right) \\
\tilde{\boldsymbol{\psi}}_{\alpha}=D^{(3)} u_{\alpha}+O(h)
\end{array}\right.
$$

An essential observation is that for all symmetric tensors $\tilde{\boldsymbol{\sigma}}_{\alpha}, \tilde{\boldsymbol{\theta}}_{\alpha}$ and $\tilde{\boldsymbol{\psi}}_{\alpha}$ of order 1,2 and 3 respectively, satisfying (2.7), the same system than (2.6) holds. For all $\beta \in \mathbb{W}_{\alpha}$ we have:

$$
\left\{\begin{array}{l}
\bar{u}_{\beta}-\bar{u}_{\alpha}=\tilde{\boldsymbol{\sigma}}_{\alpha} \bullet\left(\mathbf{z}_{\alpha \beta}^{(1)}-\mathbf{x}_{\alpha}^{(1)}\right)+\frac{1}{2} \tilde{\boldsymbol{\theta}}_{\alpha} \bullet\left(\mathbf{z}_{\alpha \beta}^{(2)}-\mathbf{x}_{\alpha}^{(2)}\right)+\frac{1}{6} \tilde{\boldsymbol{\psi}}_{\alpha} \bullet\left(\mathbf{z}_{\alpha \beta}^{(3)}-\mathbf{x}_{\alpha}^{(3)}\right)+O\left(h^{4}\right), \\
\tilde{\boldsymbol{\sigma}}_{\beta}=\tilde{\boldsymbol{\sigma}}_{\alpha}+\tilde{\boldsymbol{\theta}}_{\alpha} \bullet \mathbf{h}_{\alpha \beta}^{(1)}+\frac{1}{2} \tilde{\boldsymbol{\psi}}_{\alpha} \bullet \mathbf{h}_{\alpha \beta}^{(2)}+O\left(h^{3}\right) \\
\tilde{\boldsymbol{\theta}}_{\beta}=\tilde{\boldsymbol{\theta}}_{\alpha}+\tilde{\boldsymbol{\psi}}_{\alpha} \bullet \mathbf{h}_{\alpha \beta}^{(1)}+O\left(h^{2}\right) .
\end{array}\right.
$$

The Coupled Least Squares method consists in approximately solving (2.8), when taking out the $O\left(h^{p}\right)$ terms. Otherwise stated, we solve for $\overline{\mathfrak{u}}=\left[\bar{u}_{1}, \bar{u}_{2}, \ldots \bar{u}_{N}\right] \mapsto \tilde{\boldsymbol{\sigma}}_{\alpha}[\overline{\mathfrak{u}}], \tilde{\boldsymbol{\theta}}_{\alpha}[\overline{\mathfrak{u}}], \tilde{\boldsymbol{\psi}}_{\alpha}[\overline{\mathfrak{u}}]$ solution of the system

$$
\left\{\begin{array}{l}
\bar{u}_{\beta}-\bar{u}_{\alpha}=\tilde{\boldsymbol{\sigma}}_{\alpha}[\overline{\mathfrak{u}}] \bullet\left(\mathbf{z}_{\alpha \beta}^{(1)}-\mathbf{x}_{\alpha}^{(1)}\right)+\frac{1}{2} \tilde{\boldsymbol{\theta}}_{\alpha}[\overline{\mathfrak{u}}] \bullet\left(\mathbf{z}_{\alpha \beta}^{(2)}-\mathbf{x}_{\alpha}^{(2)}\right)+\frac{1}{6} \tilde{\boldsymbol{\psi}}_{\alpha}[\overline{\mathfrak{u}}] \bullet\left(\mathbf{z}_{\alpha \beta}^{(3)}-\mathbf{x}_{\alpha}^{(3)}\right), \quad(a), \\
\tilde{\boldsymbol{\sigma}}_{\beta}[\overline{\mathfrak{u}}]=\tilde{\boldsymbol{\sigma}}_{\alpha}[\overline{\mathfrak{u}}]+\tilde{\boldsymbol{\theta}}_{\alpha}[\overline{\mathfrak{u}}] \bullet \mathbf{h}_{\alpha \beta}^{(1)}+\frac{1}{2} \tilde{\boldsymbol{\psi}}_{\alpha}[\overline{\mathfrak{u}}] \bullet \mathbf{h}_{\alpha \beta}^{(2)}, \quad(b), \\
\tilde{\boldsymbol{\theta}}_{\beta}[\overline{\mathfrak{u}}]=\tilde{\boldsymbol{\theta}}_{\alpha}[\overline{\mathfrak{u}}]+\tilde{\boldsymbol{\psi}}_{\alpha}[\overline{\mathfrak{u}}] \bullet \mathbf{h}_{\alpha \beta}^{(1)}, \quad(c) .
\end{array}\right.
$$

Solving (2.9) raises two questions. First the linear system (2.9) does not have a solution in general. This system is rectangular with $m_{\alpha}(1+d+d(d-1) / 2)$ equations and $d+d(d+1) / 2+d(d+1)(d+2) / 6$ unknowns. For example in two dimensions and for a grid made of triangles, there are 18 equations and 9 unknowns. Therefore (2.9) is overdetermined. This leads to seek a solution in the least squares sense. Second, equations $\left(2.9_{a}\right),\left(2.9_{b}\right)$ and $\left(2.9_{c}\right)$ couple unknowns in differents cells. To overcome these problems, the following strategy is used. The system (2.9) is rewritten as:

$$
\left\{\begin{array}{l}
\mathbf{h}_{\alpha \beta} \cdot \tilde{\boldsymbol{\sigma}}_{\alpha}[\overline{\mathfrak{u}}]=\tilde{u}_{\beta}-\bar{u}_{\alpha}-\frac{1}{2} \tilde{\boldsymbol{\theta}}_{\alpha}[\overline{\mathfrak{u}}] \bullet\left(\mathbf{z}_{\alpha \beta}^{(2)}-\mathbf{x}_{\alpha}^{(2)}\right)-\frac{1}{6} \tilde{\boldsymbol{\psi}}_{\alpha}[\overline{\mathfrak{u}}] \bullet\left(\mathbf{z}_{\alpha \beta}^{(3)}-\mathbf{x}_{\alpha}^{(3)}\right), \\
\mathbf{h}_{\alpha \beta} \cdot \tilde{\boldsymbol{\theta}}_{\alpha}[\overline{\mathfrak{u}}]=\tilde{\boldsymbol{\sigma}}_{\beta}[\overline{\mathfrak{u}}]-\tilde{\boldsymbol{\sigma}}_{\alpha}[\overline{\mathfrak{u}}]-\frac{1}{2} \tilde{\boldsymbol{\psi}}_{\alpha}[\overline{\mathfrak{u}}] \bullet \mathbf{h}_{\alpha \beta}^{(2)}, \quad(b) \\
\mathbf{h}_{\alpha \beta} \cdot \tilde{\boldsymbol{\psi}}_{\alpha}[\overline{\mathfrak{u}}]=\tilde{\boldsymbol{\theta}}_{\beta}[\overline{\mathfrak{u}}]-\tilde{\boldsymbol{\theta}}_{\alpha}[\overline{\mathfrak{u}}] \quad(c) .
\end{array}\right.
$$

Let us now consider in each equation of (2.10) the unknowns in the right-hand-side as parameters. A least squares resolution is applied to each equation in (2.10). Consider first $(2.10)_{a}$. The right-hand 
side is denoted by

$$
\widetilde{\delta u}_{\alpha \beta}^{(0)}=\tilde{u}_{\beta}-\bar{u}_{\alpha}-\frac{1}{2} \tilde{\boldsymbol{\theta}}_{\alpha}[\overline{\mathfrak{u}}] \bullet\left(\mathbf{z}_{\alpha \beta}^{(2)}-\mathbf{x}_{\alpha}^{(2)}\right)-\frac{1}{6} \tilde{\boldsymbol{\psi}}_{\alpha}[\overline{\mathfrak{u}}] \bullet\left(\mathbf{z}_{\alpha \beta}^{(3)}-\mathbf{x}_{\alpha}^{(3)}\right),
$$

Therefore $(2.10)_{a}$ is rewritten as

$$
\mathbf{h}_{\alpha \beta}, \tilde{\boldsymbol{\sigma}}_{\alpha}[\overline{\mathfrak{u}}]=\widetilde{\delta u}_{\alpha \beta}^{(0)}, \quad \forall \beta \in \mathbb{W}_{\alpha} .
$$

This is a linear system of $m_{\alpha}$ equations with $d$ unknowns which are $\left[\tilde{\sigma}_{\alpha, 1}, \ldots \tilde{\sigma}_{\alpha, d}\right]$. The least squares solution gives that $\tilde{\boldsymbol{\sigma}}_{\alpha}[\overline{\mathfrak{u}}]$ is solution of

$$
\min _{\boldsymbol{\sigma}_{\alpha} \in \mathbb{R}^{d}} \sum_{\beta \in \mathbb{W}_{\alpha}}\left|{\widetilde{\delta u_{\alpha \beta}}}_{\alpha \beta}^{0}-\boldsymbol{\sigma}_{\alpha} \bullet \mathbf{h}_{\alpha}^{(1)}\right|^{2} .
$$

On the other hand, the standard least squares slope in cell $\mathcal{T}_{\alpha}$ is solution of

$$
\min _{\boldsymbol{\sigma}_{\alpha} \in \mathbb{R}^{d}} \sum_{\beta \in \mathbb{W}_{\alpha}}\left|\bar{u}_{\beta}-\bar{u}_{\alpha}-\boldsymbol{\sigma}_{\alpha} \bullet \mathbf{h}_{\alpha}^{(1)}\right|^{2}
$$

Denote $\mathcal{C}_{\alpha}$ and $\mathcal{H}_{\alpha}^{T}$ the $d \times m_{\alpha}$ matrices $\left(m_{\alpha}=\# \mathbb{W}_{\alpha}\right)$

$$
\mathcal{C}_{\alpha}=\left[\mathbf{c}_{\alpha \beta_{1}}, \mathbf{c}_{\alpha \beta_{2}}, \ldots \mathbf{c}_{\alpha \beta_{m_{\alpha}}}\right], \quad \mathcal{H}_{\alpha}^{T}=\left[\mathbf{h}_{\alpha \beta_{1}}, \mathbf{h}_{\alpha \beta_{2}}, \ldots \mathbf{h}_{\alpha \beta_{m_{\alpha}}}\right] .
$$

Assuming $\operatorname{rank}\left(\mathcal{H}_{\alpha}\right)=d$, the unique solution of (2.14) is given by the least squares operator $\mathcal{A}_{\alpha}$ defined by

$$
\mathcal{A}_{\alpha}:[\overline{\mathfrak{u}}] \mapsto \overline{\boldsymbol{\sigma}}_{\alpha}^{L S}[\overline{\mathfrak{u}}]=\sum_{\beta \in \mathbb{W}_{\alpha}} \mathbf{c}_{\alpha \beta}\left(\bar{u}_{\beta}-\bar{u}_{\alpha}\right)
$$

where

$$
\mathbf{c}_{\alpha \beta}=\left(\mathcal{H}_{\alpha}^{T} \mathcal{H}_{\alpha}\right)^{-1} \mathbf{h}_{\alpha \beta}^{(1)} \quad(=O(1 / h)) .
$$

As a result, $\tilde{\boldsymbol{\sigma}}_{\alpha}[\overline{\mathfrak{u}}]$ solution of $(2.13)$ is expressed as:

$$
\tilde{\boldsymbol{\sigma}}_{\alpha}[\overline{\mathfrak{u}}]=\overline{\boldsymbol{\sigma}}_{\alpha}^{L S}[\overline{\mathfrak{u}}]-\overline{\mathbf{a}}_{\alpha}^{(3)} \cdot \tilde{\boldsymbol{\theta}}_{\alpha}[\overline{\mathfrak{u}}]-\overline{\mathbf{b}}_{\alpha}^{(4)} \cdot \tilde{\boldsymbol{\psi}}_{\alpha}[\overline{\mathfrak{u}}] .
$$

where the two tensors $\overline{\mathbf{a}}_{\alpha}^{(3)}$ and $\overline{\mathbf{b}}_{\alpha}^{(4)}$ depend only on the grid and are given by

$$
\overline{\mathbf{a}}_{\alpha}^{(3)}=\left(\mathbf{z}_{\alpha \beta}^{(2)}-\mathbf{x}_{\alpha}^{(2)}\right) \otimes \mathbf{c}_{\alpha \beta}, \quad \overline{\mathbf{b}}_{\alpha}^{(4)}=\left(\mathbf{z}_{\alpha \beta}^{(3)}-\mathbf{x}_{\alpha}^{(3)}\right) \otimes \mathbf{c}_{\alpha \beta} .
$$

The relation (2.18) is the first relation of the Coupled Least Squares method. Note that at this stage, $\tilde{\boldsymbol{\theta}}_{\alpha}[\overline{\mathfrak{u}}]$ and $\tilde{\boldsymbol{\psi}}_{\alpha}[\overline{\mathfrak{u}}]$ are still parameters and are not determined. Consider now the least squares resolution of $(2.10)_{b}$. As before we consider $\tilde{\boldsymbol{\psi}}_{\alpha}[\overline{\mathfrak{u}}]$ in the right-hand side of $(2.10)_{b}$ as a fixed parameter. We denote

$$
\widetilde{\delta u}_{\alpha \beta}^{(1)}=\tilde{\boldsymbol{\sigma}}_{\beta}[\overline{\mathfrak{u}}]-\tilde{\boldsymbol{\sigma}}_{\alpha}[\overline{\mathfrak{u}}]-\frac{1}{2} \tilde{\boldsymbol{\psi}}_{\alpha}[\mathfrak{u}] \bullet \mathbf{h}_{\alpha \beta}^{(2)}, \quad \beta \in \mathbb{W}_{\alpha} .
$$

In $(2.10)_{b}$, the symmetric tensor $\tilde{\boldsymbol{\theta}}_{\alpha}[\overline{\mathfrak{u}}]$ is the unknown and $\widetilde{\delta u}_{\alpha \beta}^{(1)}$ is at the moment considered to be known. There are $d(d+1) / 2$ unknowns and $d m_{\alpha}$ equations. The linear system is as before overdetermined. It can be expressed as

$$
H_{\alpha}^{(2)} \tilde{\boldsymbol{\theta}}^{\alpha}[\overline{\mathfrak{u}}]=\left[\widetilde{\delta u}_{\alpha \beta}^{(1)}\right]_{\beta},
$$


where the size of the matrix $H_{\alpha}^{(2)}$ is $m_{\alpha} d \times d(d+1) / 2$. Again (2.21) is solved by least squares. This leads to

$$
\tilde{\boldsymbol{\theta}}^{\alpha}[\overline{\mathfrak{u}}]=\left(H_{\alpha}^{(2)}\right)^{+} \widetilde{\delta \mathfrak{u}}_{\alpha}^{(1)},
$$

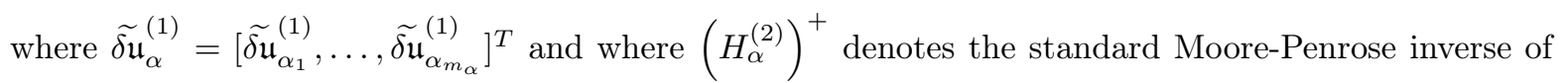
the matrix $H_{\alpha}^{(2)}$. It is defined by [10, 8]:

$$
\left(H_{\alpha}^{(2)}\right)^{+}=\left(\left(H_{\alpha}^{(2)}\right)^{T} H_{\alpha}^{(2)}\right)^{-1}\left(H_{\alpha}^{(2)}\right)^{T} .
$$

Therefore the second relation in the space of symmetric tensors of order 2 (i.e. in $\mathbb{R}^{d(d+1) / 2}$ ) is

$$
\tilde{\boldsymbol{\theta}}^{\alpha}[\overline{\mathfrak{u}}]=\left(H_{\alpha}^{(2)}\right)^{+}\left[\left(\tilde{\boldsymbol{\sigma}}_{\beta}[\overline{\mathfrak{u}}]-\tilde{\boldsymbol{\sigma}}_{\alpha}[\overline{\mathfrak{u}}]-\frac{1}{2} \tilde{\boldsymbol{\psi}}_{\alpha}[\overline{\mathfrak{u}}] \bullet \mathbf{h}_{\alpha \beta}^{(2)}\right)_{\beta}\right] .
$$

Proceeding similarly for the third relation $(2.10)_{c}$ provides a relation of the form

$$
\tilde{\boldsymbol{\psi}}^{\alpha}[\overline{\mathfrak{u}}]=\left(H_{\alpha}^{(3)}\right)^{+}\left[\left(\tilde{\boldsymbol{\theta}}_{\beta}[\overline{\mathfrak{u}}]-\tilde{\boldsymbol{\theta}}_{\alpha}[\overline{\mathfrak{u}}]\right)_{\beta}\right] .
$$

The relation (2.25) is an identity in the space of symmetric tensor of order 3 and $H_{\alpha}^{(3)}$ again is a suitable least squares matrix. The final linear system with unknowns $\tilde{\boldsymbol{\sigma}}_{\alpha}[\overline{\mathfrak{u}}], \tilde{\boldsymbol{\theta}}_{\alpha}[\overline{\mathfrak{u}}]$ and $\tilde{\boldsymbol{\psi}}_{\alpha}[\overline{\mathfrak{u}}]$ is of the form

$$
\left\{\begin{aligned}
& \tilde{\boldsymbol{\sigma}}_{\alpha}[\overline{\mathfrak{u}}]+\overline{\mathbf{a}}_{\alpha}^{(3)} \cdot \tilde{\boldsymbol{\theta}}_{\alpha}[\overline{\mathfrak{u}}]+\overline{\mathbf{b}}_{\alpha}^{(4)} \cdot \tilde{\boldsymbol{\psi}}_{\alpha}[\overline{\mathfrak{u}}]=\overline{\boldsymbol{\sigma}}_{\alpha}^{L S}[\overline{\mathfrak{u}}],(a) \\
& \tilde{\boldsymbol{\theta}}_{\alpha}[\overline{\mathfrak{u}}]+\mathbf{a}_{\alpha}^{(3)} \cdot \tilde{\boldsymbol{\psi}}_{\alpha}[\overline{\mathfrak{u}}]=\tilde{\boldsymbol{\theta}}_{\alpha}^{L S}[\overline{\mathfrak{u}}], \quad(b) \\
& \tilde{\boldsymbol{\psi}}_{\alpha}[\overline{\mathfrak{u}}]=\tilde{\boldsymbol{\psi}}_{\alpha}^{L S}[\overline{\mathfrak{u}}](c) .
\end{aligned}\right.
$$

In (2.26) we have denoted

$$
\left\{\begin{array}{c}
\tilde{\boldsymbol{\theta}}_{\alpha}^{L S}[\overline{\mathfrak{u}}]=\left(H_{\alpha}^{(2)}\right)^{+}\left[\left(\tilde{\boldsymbol{\sigma}}_{\beta}[\overline{\mathfrak{u}}]-\tilde{\boldsymbol{\sigma}}_{\alpha}[\overline{\mathrm{u}}]\right)_{\beta}\right], \\
\tilde{\boldsymbol{\psi}}_{\alpha}^{L S}[\overline{\mathfrak{u}}]=\left(H_{\alpha}^{(3)}\right)^{+}\left[\left(\tilde{\boldsymbol{\theta}}_{\beta}[\overline{\mathfrak{u}}]-\tilde{\boldsymbol{\theta}}_{\alpha}[\overline{\mathfrak{u}}]\right)_{\beta}\right] .
\end{array}\right.
$$

and where $\mathbf{a}_{\alpha}^{(3)}$ is a third tensor depending only on the grid.

Definition 2.1. The system (2.26) is called the Coupled Least Squares system for the cubic reconstruction.

Two methods to solve (2.26) can be considered. First, the Jacobi (or Gauss Seidel) iteration can be used. This approach is presented in [14]. Due to the triangular form of (2.26), a second method consists in using a forward/backward substitution method. The rest of the paper is devoted to the presentation of this second method in the particular case of the one dimensional setting. The interest of the one dimensional case is that it permits to have a better view on the algebra of the algorithm. In particular, it permits to make fully explicit the two steps of the method: first establishing the system (2.26) and second solving it. Note in addition that, even in one dimension, the reconstruction procedure on a nonequispaced grid is by no way straightforward. It clearly deserves an independent study in itself. Having solved (2.26) in one way or another, the piecewise cubic reconstruction is given by

$$
w_{\alpha}[\overline{\mathfrak{u}}](\mathbf{x})=\bar{u}_{\alpha}+\tilde{\boldsymbol{\sigma}}_{\alpha}[\overline{\mathfrak{u}}] \bullet\left[\left(\mathbf{x}-\mathbf{x}_{\alpha}\right)-\mathbf{x}_{\alpha}^{(1)}\right]+\frac{1}{2} \tilde{\boldsymbol{\theta}}_{\alpha}[\overline{\mathfrak{u}}] \bullet\left[\left(\mathbf{x}-\mathbf{x}_{\alpha}\right)^{\otimes 2}-\mathbf{x}_{\alpha}^{(2)}\right]+\frac{1}{6} \tilde{\boldsymbol{\psi}}_{\alpha}[\overline{\mathfrak{u}}] \bullet\left[\left(\mathbf{x}-\mathbf{x}_{\alpha}\right)^{\otimes 3}-\mathbf{x}_{\alpha}^{(3)}\right] .
$$


Remark 2.2. A full mathematical study in the $d$ - dimensional case, $d \geq 2$ is not completed yet. An important (and difficult) issue is to determine the geometric condition on the grid under which the matrices $\left(H_{\alpha}^{(2)}\right)^{+}$and $\left(H_{\alpha}^{(3)}\right)^{+}$do exist. In the absence of such a condition so far, we rely on numerical experiments.

\subsection{The one-dimensional case}

From now on, we consider the one dimensional case. First we give the detail of the agebra in Section 2.3 in the one dimensional case $(d=1)$. This permits to emphasize the logic of the reconstruction procedure. In this case, the calculations presented in Section 2.1 are much simpler since all tensors reduce to scalars. The neighborhood $\mathbb{W}_{\alpha}$ is $\mathbb{W}_{\alpha}=\mathbb{V}_{\alpha}^{(1)}$ with $^{3}$

$$
\mathbb{V}_{\alpha}^{(1)}=\left\{\mathcal{T}_{\alpha-1}, \mathcal{T}_{\alpha}, \mathcal{T}_{\alpha+1}\right\}
$$

The vector $h_{\alpha \beta}$ is defined by (see Fig. 1.1)

$$
h_{\alpha \beta}=x_{\beta}-x_{\alpha}, \quad \beta=\alpha-1, \alpha, \alpha+1 .
$$

In particular, $h_{\alpha \alpha}=0$. The hypothesis (8.12) on the irregular grid becomes

$$
c h \leq h_{\alpha} \leq C h, \quad c h \leq h_{\alpha \beta} \leq C h,
$$

where $c$ and $C$ are positive constants. Let $u(x)$ be a given regular function. The values $u_{\alpha}, \sigma_{\alpha}, \theta_{\alpha}$ and $\psi_{\alpha}$ are (see $\left.(2.3)\right)$ :

$$
u_{\alpha}=u\left(x_{\alpha}\right), \quad \sigma_{\alpha}=u^{\prime}\left(x_{\alpha}\right), \quad \theta_{\alpha}=u^{\prime \prime}\left(x_{\alpha}\right), \quad \psi_{\alpha}=u^{\prime \prime \prime}\left(x_{\alpha}\right) .
$$

The Taylor expansion of $u(x)$ at $x_{\alpha}$ is:

$$
u(x)=u_{\alpha}+\sigma_{\alpha}\left(x-x_{\alpha}\right)+\frac{1}{2} \theta_{\alpha}\left(x-x_{\alpha}\right)^{2}+\frac{1}{6} \psi_{\alpha}\left(x-x_{\alpha}\right)^{3}+O\left(h^{4}\right) .
$$

The average $\bar{u}_{\alpha}$ of $u(x)$ over $\mathcal{T}_{\alpha}$ satisfies:

$$
\bar{u}_{\alpha}=u_{\alpha}+\frac{\left|\mathcal{T}_{\alpha}\right|^{2}}{24} \theta_{\alpha}+O\left(h^{4}\right),
$$

Inserting $\bar{u}_{\alpha}$ in (2.33) in replacement of $u_{\alpha}$ leads to expanding $u(x)$ in the form:

$$
u(x)=\bar{u}_{\alpha}+\sigma_{\alpha}\left(x-x_{\alpha}\right)+\theta_{\alpha}\left(\frac{1}{2}\left(x-x_{\alpha}\right)^{2}-\frac{1}{24}\left|\mathcal{T}_{\alpha}\right|^{2}\right)+\frac{1}{6} \psi_{\alpha}\left(x-x_{\alpha}\right)^{3}+O\left(h^{4}\right) .
$$

This is why we look for an approximant $w_{\alpha}[\overline{\mathfrak{u}}](x)$ of $u(x)$ of the form:

$$
w_{\alpha}[\overline{\mathfrak{u}}](x)=\bar{u}_{\alpha}+\tilde{\sigma}_{\alpha}\left(x-x_{\alpha}\right)+\tilde{\theta}_{\alpha}\left(\frac{1}{2}\left(x-x_{\alpha}\right)^{2}-\frac{1}{24}\left|\mathcal{T}_{\alpha}\right|^{2}\right)+\frac{1}{6} \tilde{\psi}_{\alpha}\left(x-x_{\alpha}\right)^{3} .
$$

where $\tilde{\sigma}_{\alpha}, \tilde{\theta}_{\alpha}$ and $\tilde{\psi}_{\alpha}$ are suitable approximations to $\sigma_{\alpha}, \theta_{\alpha}$ and $\psi_{\alpha}$, respectively. Note that $(2.36)$ is the one dimensional form of the multidimensional reconstruction (2.28). Defining the reconstruction $w_{\alpha}[\overline{\mathfrak{u}}](x)$ is therefore equivalent to define $\tilde{\sigma}_{\alpha}, \tilde{\theta}_{\alpha}$ and $\tilde{\psi}_{\alpha}$ and this is the topic of the rest of the paper. In order to define a fourth order VF scheme, the reconstruction (2.36) is finally inserted in the flux function as indicated in (1.4).

\footnotetext{
${ }^{3}$ We could as well consider a priori the five points dependence stencil $\mathbb{W}_{\alpha}=\left\{\mathcal{T}_{\alpha-2}, \mathcal{T}_{\alpha-1}, \mathcal{T}_{\alpha}, \mathcal{T}_{\alpha+1}, \mathcal{T}_{\alpha+2}\right\}$
} 


\subsection{Coupled Least Squares at the continuous level}

In this section, we show how to calculate $\tilde{\sigma}_{\alpha}, \tilde{\theta}_{\alpha}$ and $\tilde{\psi}_{\alpha}$ appearing in (2.36). As already mentionned, data are located in the compact stencil $\mathbb{W}_{\alpha}=\mathbb{V}_{\alpha}^{(1)}$ only. Let us start by approximating the derivative $\sigma_{\alpha}$ by a linear relation of the form

$$
\sigma_{\alpha} \simeq \sum_{\beta \in \mathbb{W}_{\alpha}} c_{\alpha \beta}\left(\bar{u}_{\beta}-\bar{u}_{\alpha}\right)
$$

with coefficients $c_{\alpha \beta}$ to be defined. The consistency relation is

$$
\sum_{\beta \in \mathbb{W}_{\alpha}} c_{\alpha \beta} h_{\alpha \beta}=1
$$

The relation (2.38) express the property that the slope $\sigma_{0}$ of a linear function $u(x)=u_{0}+\sigma_{0} x$ is left invariant by the slope operator (2.37). Our privilegiate choice for $c_{\alpha \beta}$ in (2.38) is the least squares slope defined by

$$
\bar{\sigma}_{\alpha}^{L S}=\sum_{\beta \in \mathbb{W}_{\alpha}} c_{\alpha \beta}^{L S}\left(\bar{u}_{\beta}-\bar{u}_{\alpha}\right)
$$

where $c_{\alpha \beta}^{L S}$ is (see $\left.(2.17)\right)$,

$$
c_{\alpha \beta}^{L S}=h_{\alpha \beta} / \sum_{\beta \in \mathbb{W}_{\alpha}} h_{\alpha \beta}^{2} .
$$

The least squares slope is consistent since it satisfies (2.38). Henceforth we write $c_{\alpha \beta}$ instead of $c_{\alpha \beta}^{L S}$. Note that

$$
c_{\alpha \beta}=O(1 / h) .
$$

In the sequel, we also need the least squares values $\sigma_{\alpha}^{L S}, \theta_{\alpha}^{L S}$ and $\psi_{\alpha}^{L S}$ defined by the relations:

$$
\left\{\begin{array}{l}
\sigma_{\alpha}^{L S}=\sum_{\beta \in \mathbb{W}_{\alpha}} c_{\alpha \beta}\left(u_{\beta}-u_{\alpha}\right), \quad(a) \\
\theta_{\alpha}^{L S}=\sum_{\beta \in \mathbb{W}_{\alpha}} c_{\alpha \beta}\left(\sigma_{\beta}-\sigma_{\alpha}\right), \\
\psi_{\alpha}^{L S}=\sum_{\beta \in \mathbb{W}_{\alpha}} c_{\alpha \beta}\left(\theta_{\beta}-\theta_{\alpha}\right) . \quad(c)
\end{array}\right.
$$

Lemma 2.3. The approximate derivatives $\sigma_{\alpha}^{L S}, \theta_{\alpha}^{L S}, \psi_{\alpha}^{L S}$ in (2.42) satisfy the consistency relations

$$
\left\{\begin{array}{l}
\sigma_{\alpha}+a_{\alpha} \theta_{\alpha}+b_{\alpha} \psi_{\alpha}=\sigma_{\alpha}^{L S}+O\left(h^{3}\right), \\
\theta_{\alpha}+a_{\alpha} \psi_{\alpha}=\theta_{\alpha}^{L S}+O\left(h^{2}\right), \quad(b) \\
\psi_{\alpha}=\psi_{\alpha}^{L S}+O(h), \quad(c)
\end{array}\right.
$$

with

$$
\left\{\begin{array}{l}
\sigma_{\alpha}=u^{\prime}\left(x_{\alpha}\right), \\
\theta_{\alpha}=u^{\prime \prime}\left(x_{\alpha}\right), \\
\psi_{\alpha}=u^{\prime \prime \prime}\left(x_{\alpha}\right) .
\end{array}\right.
$$

Using the l-momentum $H_{\alpha}^{(l)}$ in $\mathcal{T}_{\alpha}$ defined by:

$$
H_{\alpha}^{(l)}=\sum_{\beta \in \mathbb{W}_{\alpha}} h_{\alpha \beta}^{l},
$$


the coefficients $a_{\alpha}, b_{\alpha}$ in (2.43) are:

$$
a_{\alpha}=\frac{1}{2} \frac{H_{\alpha}^{(3)}}{H_{\alpha}^{(2)}}, \quad b_{\alpha}=\frac{1}{6} \frac{H_{\alpha}^{(4)}}{H_{\alpha}^{(2)}} .
$$

Furthermore, replacing in (2.43) a the pointwise least squares value $\sigma_{\alpha}^{L S}$ by $\bar{\sigma}_{\alpha}^{L S}$, gives

$$
\sigma_{\alpha}+\bar{a}_{\alpha} \theta_{\alpha}+\bar{b}_{\alpha} \psi_{\alpha}=\bar{\sigma}_{\alpha}^{L S}+O\left(h^{3}\right)
$$

where the modified coefficients $\bar{a}_{\alpha}$ and $\bar{b}_{\alpha}$ are

$$
\bar{a}_{\alpha}=a_{\alpha}+\frac{1}{24} \sum_{\beta \in \mathbb{W}_{\alpha}} c_{\alpha \beta}\left(\left|\mathcal{T}_{\beta}\right|^{2}-\left|\mathcal{T}_{\alpha}\right|^{2}\right) \text { and } \bar{b}_{\alpha}=b_{\alpha}+\frac{1}{24} \sum_{\beta \in \mathbb{W}_{\alpha}} c_{\alpha \beta}\left|\mathcal{T}_{\beta}\right|^{2} h_{\alpha \beta} .
$$

Proof. For all cells $\mathcal{T}_{\beta} \in \mathbb{W}_{\alpha}$, the Taylor expansion of $u\left(x_{\beta}\right)-u\left(x_{\alpha}\right)$ is

$$
u_{\beta}-u_{\alpha}=\sigma_{\alpha} h_{\alpha \beta}+\frac{1}{2} \theta_{\alpha} h_{\alpha \beta}^{2}+\frac{1}{6} \psi_{\alpha} h_{\alpha \beta}^{3}+O\left(h^{4}\right) .
$$

Therefore the slope $\sigma_{\alpha}^{L S}$ in $(2.42)_{a}$ satisfies

$$
\sigma_{\alpha}^{L S}=\sum_{\beta \in \mathbb{W}_{\alpha}} c_{\alpha \beta}\left(\sigma_{\alpha} h_{\alpha \beta}+\frac{1}{2} \theta_{\alpha} h_{\alpha \beta}^{2}+\frac{1}{6} \psi_{\alpha} h_{\alpha \beta}^{3}\right)+O\left(h^{3}\right) .
$$

Using (2.40) and (2.38), the identity (2.50) becomes $(2.43)_{a}$ where $a_{\alpha}$ and $b_{\alpha}$ are defined in (2.46). The relations $(2.43)_{b c}$ are obtained in the same way. We now prove (2.47). We deduce from (2.34), (2.42) and (2.49) that (note that $\left|\mathcal{T}_{\alpha}\right|^{2}=O\left(h^{2}\right)$ ),

$$
\begin{aligned}
\sigma_{\alpha}^{L S} & =\sum_{\beta \in \mathbb{W}_{\alpha}} c_{\alpha \beta}\left(\bar{u}_{\beta}-\bar{u}_{\alpha}\right)-\frac{1}{24} \sum_{\beta \in \mathbb{W}_{\alpha}} c_{\alpha \beta}\left(\left|\mathcal{T}_{\beta}\right|^{2} \theta_{\beta}-\left|\mathcal{T}_{\alpha}\right| \theta_{\alpha}^{2}\right)+O\left(h^{4}\right) \\
& =\bar{\sigma}_{\alpha}^{L S}-\frac{1}{24} \sum_{\beta \in \mathbb{W}_{\alpha}} c_{\alpha \beta}\left(\left|\mathcal{T}_{\beta}\right|^{2}\left(\theta_{\alpha}+h_{\alpha \beta} \psi_{\alpha}\right)-\left|\mathcal{T}_{\alpha}\right|^{2} \theta_{\alpha}\right)+O\left(h^{4}\right) \\
& =\bar{\sigma}_{\alpha}^{L S}-\frac{\theta_{\alpha}}{24}\left(\sum_{\beta \in \mathbb{W}_{\alpha}} c_{\alpha \beta}\left(\left|\mathcal{T}_{\beta}\right|^{2}-\left|\mathcal{T}_{\alpha}\right|^{2}\right)\right) \\
& -\frac{\psi_{\alpha}}{24}\left(\sum_{\beta \in \mathbb{W}_{\alpha}} c_{\alpha \beta}\left|\mathcal{T}_{\beta}\right|^{2} h_{\alpha \beta}\right)+O\left(h^{4}\right) .
\end{aligned}
$$

Replacing in the preceding relation $\sigma_{\alpha}^{L S}$ by $\left(\right.$ see $\left.(2.43)_{a}\right)$

$$
\sigma_{\alpha}^{L S}=\sigma_{\alpha}+a_{\alpha} \theta_{\alpha}+b_{\alpha} \psi_{\alpha}+O\left(h^{3}\right)
$$

we deduce that

$$
\begin{aligned}
\sigma_{\alpha} & +\theta_{\alpha}\left(a_{\alpha}+\frac{1}{24} \sum_{\beta \in \mathbb{W}_{\alpha}} c_{\alpha \beta}\left(\left|\mathcal{T}_{\beta}\right|^{2}-\left|\mathcal{T}_{\alpha}\right|^{2}\right)\right)+\psi_{\alpha}\left(b_{\alpha}+\frac{1}{24} \sum_{\beta \in \mathbb{W}_{\alpha}} c_{\alpha \beta}\left|\mathcal{T}_{\beta}\right|^{2} h_{\alpha \beta}\right) \\
& =\bar{\sigma}_{\alpha}^{L S}+O\left(h^{3}\right) .
\end{aligned}
$$

This proves (2.47), with the coefficients $\bar{a}_{\alpha}, \bar{b}_{\alpha}$ given in (2.48). 


\section{A Cubic Reconstruction Procedure}

In this section the Coupled Least Squares resolution procedure is detailed. We show how to use Lemma 2.3 to define our cubic reconstruction. Let us start from the relations (2.47) and $(2.43)_{b c}$ above relating $\sigma_{\alpha}, \theta_{\alpha}, \psi_{\alpha}, \bar{\sigma}_{\alpha}^{L S}, \theta_{\alpha}^{L S}$ and $\psi_{\alpha}^{L S}$. These relations are, (compare (2.26)):

$$
\left\{\begin{array}{l}
\sigma_{\alpha}+\bar{a}_{\alpha} \theta_{\alpha}+\bar{b}_{\alpha} \psi_{\alpha}=\bar{\sigma}_{\alpha}^{L S}+O\left(h^{3}\right), \\
\theta_{\alpha}+a_{\alpha} \psi_{\alpha}=\theta_{\alpha}^{L S}+O\left(h^{2}\right), \quad(b) \\
\psi_{\alpha}=\psi_{\alpha}^{L S}+O(h) . \quad(c)
\end{array}\right.
$$

Recall that $\sigma_{\alpha}, \theta_{\alpha}$ and $\psi_{\alpha}$ denote the exact derivatives $u^{\prime}\left(x_{\alpha}\right), u^{\prime \prime}\left(x_{\alpha}\right)$ and $u^{\prime \prime \prime}\left(x_{\alpha}\right)$, respectively. However, consider for a moment the system (3.1) as a $3 \times 3$ linear system with $\sigma_{\alpha}, \theta_{\alpha}$ and $\psi_{\alpha}$ as "unknowns". A forward-backward "resolution" provides Taylor expansions of $\sigma_{\alpha}, \theta_{\alpha}$ and $\psi_{\alpha}$ in terms of the value $\bar{\sigma}_{\beta}^{L S}$. This is expressed in the following

Proposition 3.1. The exact derivatives $u^{\prime}\left(x_{\alpha}\right)=\sigma_{\alpha}, u^{\prime \prime}\left(x_{\alpha}\right)=\theta_{\alpha}, u^{\prime \prime \prime}\left(x_{\alpha}\right)=\psi_{\alpha}$ satisfy the relations

$$
\left\{\begin{array}{l}
\psi_{\alpha}=\sum_{\beta \in \mathbb{W}_{\alpha}} c_{\alpha \beta}^{*}\left(\sum_{\gamma \in \mathcal{V}_{\beta}} \tilde{c}_{\beta \gamma}\left(\bar{\sigma}_{\gamma}^{L S}-\bar{\sigma}_{\beta}^{L S}\right)-\sum_{\delta \in \mathbb{W}_{\alpha}} \tilde{c}_{\alpha \delta}\left(\bar{\sigma}_{\delta}^{L S}-\bar{\sigma}_{\alpha}^{L S}\right)\right)+O(h), \\
\theta_{\alpha}=-\tilde{a}_{\alpha} \psi_{\alpha}+\sum_{\beta \in \mathcal{V}_{\alpha}} \tilde{c}_{\alpha \beta}\left(\bar{\sigma}_{\beta}^{L S}-\bar{\sigma}_{\alpha}^{L S}\right)+O\left(h^{2}\right), \quad(b) \\
\sigma_{\alpha}=-\bar{a}_{\alpha} \theta_{\alpha}-\bar{b}_{\alpha} \psi_{\alpha}+\bar{\sigma}_{\alpha}^{L S}+O\left(h^{3}\right), \quad(c)
\end{array}\right.
$$

where

- The coefficients $\bar{a}_{\alpha}$ and $\bar{b}_{\alpha}$ are given in (2.48),

- The coefficients $\tilde{a}_{\alpha}$ and $\tilde{c}_{\alpha \beta}$ are defined by:

$$
\tilde{a}_{\alpha}=\frac{a_{\alpha}+\sum_{\beta \in \mathbb{W}_{\alpha}} c_{\alpha \beta}\left(\bar{b}_{\beta}-\bar{b}_{\alpha}+\bar{a}_{\beta} h_{\alpha \beta}\right)}{1+\sum_{\beta \in \mathbb{W}_{\alpha}} c_{\alpha \beta}\left(\bar{a}_{\beta}-\bar{a}_{\alpha}\right)},
$$

and

$$
\tilde{c}_{\alpha \beta}=\frac{c_{\alpha \beta}}{1+\sum_{\beta \in \mathbb{W}_{\alpha}} c_{\alpha \beta}\left(\bar{a}_{\beta}-\bar{a}_{\alpha}\right)} .
$$

- The coefficient $c_{\alpha \beta}^{*}$ is given by

$$
c_{\alpha \beta}^{*}=\frac{c_{\alpha \beta}}{1+\sum_{\delta \in \mathbb{W}_{\alpha}} c_{\alpha \delta}\left(\tilde{a}_{\delta}-\tilde{a}_{\alpha}\right)} .
$$

Proof. First note that Equation $(3.2)_{(c)}$ is identical to Equation $(3.1)_{(a)}$ and therefore there is nothing to prove. Second, consider the proof of $(3.2)_{(b)}$. The relation $(3.1)_{(b)}$ is

$$
\theta_{\alpha}+a_{\alpha} \psi_{\alpha}=\theta_{\alpha}^{L S}+O\left(h^{2}\right)
$$

Replacing in the right-hand side $\theta_{\alpha}^{L S}$ by its value in $(2.42)_{(b)}$ gives

$$
\theta_{\alpha}+a_{\alpha} \psi_{\alpha}=\sum_{\beta \in \mathbb{W}_{\alpha}} c_{\alpha \beta}\left(\sigma_{\beta}-\sigma_{\alpha}\right)+O\left(h^{2}\right) .
$$


Consider now the equation (2.47) in all cells $\mathcal{T}_{\beta}$

$$
\sigma_{\beta}+\bar{a}_{\beta} \theta_{\beta}+\bar{b}_{\beta} \psi_{\beta}=\bar{\sigma}_{\beta}^{L S}+O\left(h^{3}\right) .
$$

For all cells $\mathcal{T}_{\beta}$ close to $\mathcal{T}_{\alpha}$, a Taylor expansion gives

$$
\left\{\begin{array}{l}
\theta_{\beta}=\theta_{\alpha}+h_{\alpha \beta} \psi_{\alpha}+O\left(h^{2}\right), \quad(a) \\
\psi_{\beta}=\psi_{\alpha}+O(h)
\end{array}\right.
$$

Replacing in (3.8) the values of $\theta_{\beta}$ and $\psi_{\beta}$ given in (3.9) yields (note that $\bar{a}_{\alpha}=O(h)$ and $\bar{b}_{\alpha}=O\left(h^{2}\right)$ )

$$
\sigma_{\beta}+\bar{a}_{\beta}\left(\theta_{\alpha}+h_{\alpha \beta} \psi_{\alpha}\right)+\bar{b}_{\beta} \psi_{\alpha}=\bar{\sigma}_{\beta}^{L S}+O\left(h^{3}\right),
$$

or equivalently

$$
\sigma_{\beta}=\bar{\sigma}_{\beta}^{L S}-\bar{a}_{\beta} \theta_{\alpha}-\left(\bar{a}_{\beta} h_{\alpha \beta}+\bar{b}_{\beta}\right) \psi_{\alpha}+O\left(h^{3}\right) .
$$

Inserting finally (3.11) in (3.8) gives

$$
\begin{aligned}
\theta_{\alpha}+a_{\alpha} \psi_{\alpha} & =\sum_{\beta \in \mathbb{W}_{\alpha}} c_{\alpha \beta}\left(\bar{\sigma}_{\alpha}^{L S}-\bar{a}_{\beta} \theta_{\alpha}-\left(\bar{a}_{\beta} h_{\alpha \beta}+\bar{b}_{\beta}\right) \psi_{\alpha}-\sigma_{\alpha}\right)+O\left(h^{2}\right) \\
& =\sum_{\beta \in \mathbb{W}_{\alpha}} c_{\alpha \beta}\left(\bar{\sigma}_{\beta}^{L S}-\bar{a}_{\beta} \theta_{\alpha}-\left(\bar{a}_{\beta} h_{\alpha \beta}+\bar{b}_{\beta}\right) \psi_{\alpha}-\bar{\sigma}_{\alpha}^{L S}+\bar{a}_{\alpha} \theta_{\alpha}+\bar{b}_{\alpha} \psi_{\alpha}\right) \\
& +O\left(h^{2}\right) .
\end{aligned}
$$

Collecting the terms in $\theta_{\alpha}$ and $\psi_{\alpha}$ in the left-hand-side gives

$$
\begin{aligned}
\left(1+\sum c_{\alpha \beta}\left(\bar{a}_{\beta}-\bar{a}_{\alpha}\right)\right) \theta_{\alpha} & +\left(a_{\alpha}+\sum_{\beta} c_{\alpha \beta}\left(\bar{b}_{\beta}-\bar{b}_{\alpha}+\bar{a}_{\beta} h_{\alpha \beta}\right)\right) \psi_{\alpha} \\
& =\sum_{\beta \in \mathbb{W}_{\alpha}} c_{\alpha \beta}\left(\bar{\sigma}_{\beta}^{L S}-\bar{\sigma}_{\alpha}^{L S}\right)+O\left(h^{2}\right) .
\end{aligned}
$$

Dividing each side by the coefficient

$$
1+\sum_{\beta \in \mathbb{W}_{\alpha}} c_{\alpha \beta}\left(\bar{a}_{\beta}-\bar{a}_{\alpha}\right)
$$

this is rewritten as

$$
\theta_{\alpha}+\tilde{a}_{\alpha} \psi_{\alpha}=\sum_{\beta \in \mathbb{W}_{\alpha}} \tilde{c}_{\alpha \beta}\left(\bar{\sigma}_{\beta}^{L S}-\bar{\sigma}_{\alpha}^{L S}\right)+O\left(h^{2}\right),
$$

where $\tilde{a}_{\alpha}, \tilde{c}_{\alpha \beta}$ are given in (3.4). Therefore $(3.2)_{(b)}$ is proved and we prove $(3.2)_{(c)}$ in a similar way. Using (3.13) at $x_{\beta}$ and the relation $\psi_{\beta}=\psi_{\alpha}+O(h)$ gives the following expression for $\theta_{\beta}$

$$
\theta_{\beta}=-\tilde{a}_{\beta} \psi_{\alpha}+\sum_{\gamma \in \mathbb{W}_{\beta}} \tilde{c}_{\beta \gamma}\left(\bar{\sigma}_{\gamma}^{L S}-\bar{\sigma}_{\beta}^{L S}\right)+O\left(h^{2}\right) .
$$


The identity $(2.42)_{(c)}$ is expressed using $(3.1),(3.14)$ and $(3.9)_{(b)}$ as

$$
\begin{aligned}
\psi_{\alpha} & =\sum_{\beta \in \mathbb{W}_{\alpha}} c_{\alpha \beta}\left(\theta_{\beta}-\theta_{\alpha}\right) \\
& =\sum_{\beta \in \mathbb{W}_{\alpha}} c_{\alpha \beta}\left(-\tilde{a}_{\beta} \psi_{\beta}+\sum_{\gamma \in \mathbb{W}_{\beta}} \tilde{c}_{\beta \gamma}\left(\bar{\sigma}_{\gamma}^{L S}-\bar{\sigma}_{\beta}^{L S}\right)-\theta_{\alpha}\right)+O(h) \\
& =\sum_{\beta \in \mathbb{W}_{\alpha}} c_{\alpha \beta}\left(\tilde{a}_{\alpha} \psi_{\alpha}-\tilde{a}_{\beta} \psi_{\beta}+\sum_{\gamma \in \mathbb{W}_{\beta}} \tilde{c}_{\beta \gamma}\left(\bar{\sigma}_{\gamma}^{L S}-\bar{\sigma}_{\beta}^{L S}\right)\right. \\
& \left.-\sum_{\delta \in \mathbb{W}_{\alpha}} \tilde{c}_{\alpha \delta}\left(\bar{\sigma}_{\delta}^{L S}-\bar{\sigma}_{\alpha}^{L S}\right)\right)+O(h) \\
& =\psi_{\alpha}\left(-\sum_{\beta \in \mathbb{W}_{\alpha}} c_{\alpha \beta}\left(\tilde{a}_{\beta}-\tilde{a}_{\alpha}\right)\right) \\
& +\sum_{\beta \in \mathbb{W}_{\alpha}} c_{\alpha \beta}\left(\sum_{\gamma \in \mathbb{W}_{\beta}} \tilde{c}_{\beta \gamma}\left(\bar{\sigma}_{\gamma}^{L S}-\sigma_{\beta}^{L S}\right)-\sum_{\delta \in \mathbb{W}_{\alpha}} \tilde{c}_{\alpha \delta}\left(\bar{\sigma}_{\delta}^{L S}-\bar{\sigma}_{\alpha}^{L S}\right)\right)+O(h) .
\end{aligned}
$$

Collecting in the left hand side all the terms in $\psi_{\alpha}$ gives:

$$
\begin{aligned}
\left(1+\sum_{\delta \in \mathbb{W}_{\alpha}} c_{\alpha \delta}\left(\tilde{a}_{\delta}-\tilde{a}_{\alpha}\right)\right) \psi_{\alpha} & =\sum_{\beta \in \mathbb{W}_{\alpha}} c_{\alpha \beta}\left(\sum_{\gamma \in \mathbb{W}_{\beta}} \tilde{c}_{\beta \gamma}\left(\bar{\sigma}_{\gamma}^{L S}-\bar{\sigma}_{\beta}^{L S}\right)\right. \\
& \left.-\sum_{\delta \in \mathbb{W}_{\alpha}} \tilde{c}_{\alpha \delta}\left(\bar{\sigma}_{\delta}^{L S}-\bar{\sigma}_{\alpha}^{L S}\right)\right)+O(h) .
\end{aligned}
$$

Dividing by the coefficient $\left(1+\sum_{\delta} c_{\alpha \delta}\left(\tilde{a}_{\delta}-\tilde{a}_{\alpha}\right)\right)$ yields $(3.2)_{(a)}$ where $c_{\alpha \beta}^{*}$ is given in (3.5) and the proof is complete.

Finally we define the quantities $\tilde{\psi}_{\alpha}, \tilde{\theta}_{\alpha}$ and $\tilde{\sigma}_{\alpha}$ by droping in the relations $(3.2)$ the $O\left(h^{p}\right)$ terms. This suggests the following

Corollary 3.2. Let us define the values $\tilde{\psi}_{\alpha}, \tilde{\theta}_{\alpha}$ and $\tilde{\sigma}_{\alpha}$ by

$$
\left\{\begin{array}{l}
\tilde{\psi}_{\alpha}=\sum_{\beta \in \mathbb{W}_{\alpha}} c_{\alpha \beta}^{*}\left(\sum_{\gamma \in \mathbb{W}_{\beta}} \tilde{c}_{\beta \gamma}\left(\bar{\sigma}_{\gamma}^{L S}-\bar{\sigma}_{\beta}^{L S}\right)-\sum_{\delta \in \mathcal{V}_{\alpha}} \tilde{c}_{\alpha \delta}\left(\bar{\sigma}_{\delta}^{L S}-\bar{\sigma}_{\alpha}^{L S}\right)\right) \\
\tilde{\theta}_{\alpha}=-\tilde{a}_{\alpha} \tilde{\psi}_{\alpha}+\sum_{\beta \in \mathbb{W}_{\alpha}} \tilde{c}_{\alpha \beta}\left(\bar{\sigma}_{\beta}^{L S}-\bar{\sigma}_{\alpha}^{L S}\right), \\
\tilde{\sigma}_{\alpha}=-\bar{a}_{\alpha} \tilde{\theta}_{\alpha}-\bar{b}_{\alpha} \tilde{\psi}_{\alpha}+\bar{\sigma}_{\alpha}^{L S} . \quad(c)
\end{array}\right.
$$

These three values are approximations to $\psi_{\alpha}, \theta_{\alpha}$ and $\sigma_{\alpha}$ respectively with the following accuracy:

$$
\begin{cases}\tilde{\psi}_{\alpha}=\psi_{\alpha}+O(h), & (a) \\ \tilde{\theta}_{\alpha}=\theta_{\alpha}+O\left(h^{2}\right), & (b) \\ \tilde{\sigma}_{\alpha}=\sigma_{\alpha}+O\left(h^{3}\right) . & (c)\end{cases}
$$

Proof. The consistency relations (3.16) result directly from comparing (3.15) and (3.2) and from the fact that $a_{\alpha}=O(h), \tilde{a}_{\alpha}=O(h), \bar{a}_{\alpha}=O(h)$ and $\bar{b}_{\alpha}=O\left(h^{2}\right)$. 
The outcome of the preceding analysis is the following algorithm to calculate $\tilde{\psi}_{\alpha}, \tilde{\theta}_{\alpha}$ and $\tilde{\sigma}_{\alpha}$. Suppose given a function $u(x)$ with averages in the cells $\mathcal{T}_{\alpha}$ given by $\overline{\mathfrak{u}}=\left[\bar{u}_{1}, \bar{u}_{2}, \ldots, \bar{u}_{N}\right]$. The relations (3.15) translate in the following computational procedure:

Algorithm 1. 1. Using (2.39), compute $\bar{\sigma}_{\alpha}^{L S}$ in terms of the averages $\bar{u}_{\alpha}$ for all $\alpha$.

2. Compute $\sum_{\beta \in \mathbb{W}_{\alpha}} \tilde{c}_{\alpha \beta}\left(\bar{\sigma}_{\beta}^{L S}-\bar{\sigma}_{\alpha}^{L S}\right)$ with $\tilde{c}_{\alpha \beta}$ given in (3.4), for all $\alpha$.

3. Using $(3.15)_{(a)}$, compute the approximate third order derivative $\tilde{\psi}_{\alpha}$ for all $\alpha$.

4. Using $(3.15)_{(b)}$, compute the second order derivative $\tilde{\theta}_{\alpha}$ for all $\alpha$.

5. Using $(3.15)_{(c)}$, compute the first order derivative $\tilde{\sigma}_{\alpha}$ for all $\alpha$.

Our cubic reconstruction has finally the following form:

Proposition 3.3. Let $\overline{\mathfrak{u}}=\left[\bar{u}_{1}, \bar{u}_{2}, \ldots, \bar{u}_{N}\right]$ be the vector of the averages of a given function $u(x)$ over the cells $\mathcal{T}_{\alpha}$ of the grid. Then the cubic reconstruction polynomial defined by (2.36) is a fourth order accurate reconstruction of $u(x)$ near $\mathcal{T}_{\alpha}$.

Proof. This is a simple consequence of (2.35).

Remark 3.4. The difficulty mentioned in Remark 2.2 does not exist here, since the system (2.43) (droping the $O\left(h^{p}\right)$ terms) consists of 3 equations with 3 unknowns $\tilde{\sigma}_{\alpha}, \tilde{\theta}_{\alpha}$ and $\tilde{\psi}_{\alpha}$. Note however that the logic of the method is the same in the one-dimensional and the multidimensional cases.

Remark 3.5. The approximations $\tilde{\sigma}_{\alpha}, \tilde{\theta}_{\alpha}$ and $\tilde{\psi}_{\alpha}$ depend by construction on data located in cells $\mathcal{T}_{\beta}$ with $\beta \in\{\alpha-3, \alpha-2, \alpha-1, \alpha, \alpha+1, \alpha+2, \alpha+3\}$. This dependence stencil of the reconstruction is not the accessed stencil at each step of Algorithm 1, which is the compact stencil $\mathbb{W}_{\alpha}=\left\{\mathcal{T}_{\alpha-1}, \mathcal{T}_{\alpha}, \mathcal{T}_{\alpha+1}\right\}$.

Remark 3.6. The preceding analysis does not apply at boundary cells since the neighborood set $\mathcal{V}_{\alpha}$ for such cells is not complete. The question of defining a fourth order reconstruction at boundary cells is difficult. It may require one-sided approximations. We do not elaborate further on this point in this paper. In practice, second-order reconstruction is used in boundary cells.

\section{A Fourth Order Finite Volume method}

\subsection{Semi-discrete in space approximation}

Let us come back to the conservation law (1.1)

$$
\partial_{t} u+\partial_{x} f(u)=0 .
$$

The semi-discrete scheme in $(1.2)$ is

$$
\frac{d v_{\alpha}(t)}{d t}=-\frac{1}{\left|\mathcal{T}_{\alpha}\right|}\left[f_{\alpha+1 / 2}(t)-f_{\alpha-1 / 2}(t)\right], \quad 1 \leq \alpha \leq N
$$

where

- The vector $V(t)=\left[v_{1}(t), v_{2}(t), \cdots, v_{N}(t)\right]^{T} \in \mathbb{R}^{N}$ has components $v_{\alpha}(t)$ defined by:

$$
v_{\alpha}(t) \simeq \bar{u}_{\alpha}(t)=\frac{1}{\left|\mathcal{T}_{\alpha}\right|} \int_{\mathcal{T}_{\alpha}} u(x, t) d x, \quad 1 \leq \alpha \leq N,
$$

and where $u(x, t)$ is the solution of (4.1). 
- The reconstruction operator $V=\left[v_{1}, v_{2}, \ldots, v_{N}\right] \mapsto w[V]_{\alpha}(x)$ is given by $(2.36)$.

- The numerical flux function is denoted by $\left(u_{L}, u_{R}\right) \mapsto F\left(u_{L}, u_{R}\right)$.

Consider the particular case of the linear convection equation

$$
\partial_{t} u+c \partial_{x} u=0, \quad c>0, \quad x \in[0,1],
$$

with periodic boundary conditions. In this case, the function $t \mapsto \bar{u}_{\alpha}(t)$ satisfies the equation:

$$
\frac{d \bar{u}_{\alpha}(t)}{d t}=-\frac{c}{\left|\mathcal{T}_{\alpha}\right|}\left(u\left(x_{\alpha, \alpha+1}, t\right)-u\left(x_{\alpha-1, \alpha}, t\right)\right)
$$

In this case, the semi-discrete scheme (4.2) is:

$$
\frac{d v_{\alpha}}{d t}=-\frac{c}{\left|\mathcal{T}_{\alpha}\right|}\left(w_{\alpha}[V(t)]\left(x_{\alpha, \alpha+1}\right)-w_{\alpha-1}[V(t)]\left(x_{\alpha-1, \alpha}\right)\right) .
$$

where the polynomial $w_{\alpha}[V](x)$ in (2.36) is calculated by the Algorithm 1 in Section 3. In matrix form, the scheme (4.6) is expressed as the linear dynamical system:

$$
\frac{d}{d t} V(t)=\mathbf{J} V(t), \quad \mathbf{J} \in \mathbb{M}_{N}(\mathbb{R}),
$$

with $V(t)=\left[v_{1}(t), v_{2}(t) \cdots, v_{N}(t)\right]^{T} \in \mathbb{R}^{N}$. The coefficients $J_{\alpha \beta}$ of $\mathbf{J}$ are such that:

$$
\sum_{\beta=1}^{N} J_{\alpha \beta} V_{\beta}=-\frac{c}{\left|\mathcal{T}_{\alpha}\right|}\left(w_{\alpha}[V]\left(x_{\alpha, \alpha+1}\right)-w_{\alpha-1}[V]\left(x_{\alpha-1, \alpha}\right)\right) .
$$

Note that a proof of the fourth order accuracy in space of the scheme (4.6) is by no way easy in the case of a general irregular grid. In the case of a regular grid, we have the following

Proposition 4.1. On a regular grid with stepsize $h$, the scheme (4.6) is fourth order accurate with respect to the equation

$$
\frac{d \bar{u}_{\alpha}(t)}{d t}=-\frac{c}{h}\left(u\left(x_{\alpha, \alpha+1}, t\right)-u\left(x_{\alpha-1, \alpha}, t\right)\right) .
$$

Proof. We refer to the Appendix for a proof. Further comments in the particular case of an irregular grid are also given.

\subsection{Statistical analysis for linear stability}

An important question concerning the reconstruction (2.36) is whether it provides stability of the linear system (4.7). This is related to the location of the spectrum of the matrix $\mathbf{J}$ in the complex plane. Due to the irregularity of the grid, this spectrum is not analytically known in general, and therefore we must rely on some numerical evaluation. We refer to [15] for the same stability problem in three dimensions and in the case of a piecewise linear reconstruction,

Consider a periodic grid with cells of length $\left|\mathcal{T}_{\alpha}\right|=\kappa_{\alpha} h$. The coefficient $\kappa_{\alpha}$ stands for a measure of the irregularity of the grid. The $\kappa_{\alpha}$ satisfy $\sum_{\alpha} \kappa_{\alpha}=1$. The spectral abscissa of $\mathbf{J}$ is defined by:

$$
\Lambda=\max _{1 \leq k \leq N} \operatorname{Re}\left(\lambda_{k}\right)
$$



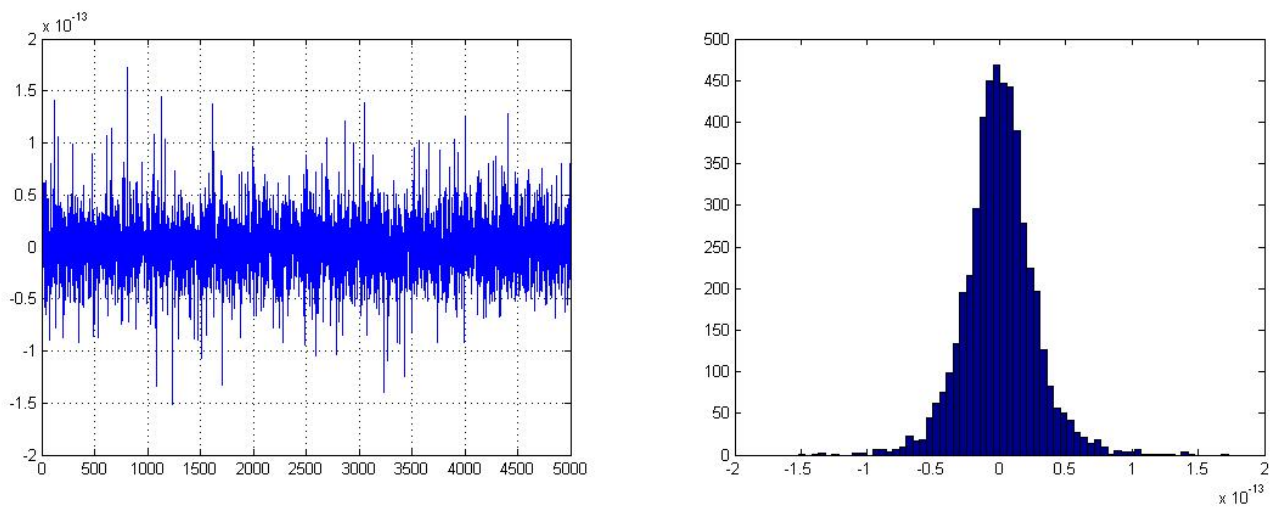

Figure 4.1: Left panel: spectral abscissa $\Lambda$ (4.10) for the scheme (4.6) for a sample of 5000 random irregular grids of size 64 . The scale along $y$ is $10^{-13}$. Right panel: distribution of the spectral absissa $\Lambda$ for a sample of 5000 random grids of size 64: a Gaussian distribution around the value 0 is clearly observed. This indicates a stable scheme.

The dynamical system (4.7) is stable when $\Lambda \leq 0$. We adopt here an elementary statistical point of view. The spectral absissa $\Lambda$ is evaluated using a sample of irregular grids. Each grid of the sample has $N$ cells. The irregularity factor $\kappa_{\alpha}, 1 \leq \alpha \leq N$, is randomly selected so that

$$
\kappa_{\alpha} \sim 1+C r_{\alpha}>0
$$

where $C \in[0,1)$ is a constant and $r=\left[r_{\alpha}\right] \in[-1,1]^{N}$ is a random array of size $N$. The magnitude of the constant $C$ determines the irregularity of the grid. Testing a large sample of such grids provides a statistical evaluation of the value of the spectral abscissa $\Lambda$. Doing so, no instability was observed, to computer accuracy. On Fig. 4.1 (left panel), we display the spectral abscissa as a function of the index $k$ of 5000 randomly selected grids of size $N=64$. This was also performed (not shown) using a sample of 1000 grids of size $N=32$. In both cases, the irregularity constant in (4.11) was choosen as $C=0.99$, which corresponds to highly irregular grids. As can be observed, $\Lambda \simeq 0$ to computer accuracy. On Fig. 4.1 (right panel) the distribution of $\Lambda$ is represented, which appears to be Gaussian. Furthermore a T-test at the confidence level $\alpha=0.05$ with null hypothesis on the mean value $\bar{\Lambda}=0$ (vs $\bar{\Lambda} \neq 0$ ) provides a non-rejection and a confidence interval of $\left[\Lambda_{\min }, \Lambda_{\max }\right]=[-0.0085(-14), 0.14(-14)]($ for $\bar{\Lambda})$. This clearly indicates that the eigenvalues of the matrix $\mathbf{J}$ are located in the left complex half-plane and therefore we can infer that that the linear dynamical system (4.6) is stable.

Note that due to the irregularity of the grid, a theoretical localization of $\Lambda$ seems a difficult problem of spectral analysis.

\subsection{Dissipation and dispersion analysis}

In the particular case of a regular grid with step-size $h$, the approximation (4.6) can be analytically derived. The coefficients $H_{\alpha}^{(2)}, H_{\alpha}^{(3)}$ and $H_{\alpha}^{(4)}$ in $(2.45)$ are

$$
H_{\alpha}^{(2)}=2 h^{2}, \quad H_{\alpha}^{(3)}=0, \quad H_{\alpha}^{(4)}=2 h^{4} .
$$

The coefficients $a_{\alpha}, \tilde{a}_{\alpha}, \bar{a}_{\alpha}, b_{\alpha}, \bar{b}_{\alpha}$ in (2.46) are

$$
a_{\alpha}=\tilde{a}_{\alpha}=\bar{a}_{\alpha}=0, \quad b_{\alpha}=\frac{h^{2}}{6}, \bar{b}_{\alpha}=\frac{5}{24} h^{2} .
$$


The coefficients $c_{\alpha, \alpha+1}, c_{\alpha, \alpha-1}, c_{\alpha, \alpha+1}^{*}, c_{\alpha, \alpha-1}^{*}$ in (2.42) and (3.5) are

$$
c_{\alpha, \alpha+1}=\frac{1}{2 h}, \quad c_{\alpha, \alpha-1}=-\frac{1}{2 h}, \quad c_{\alpha, \alpha+1}^{*}=\frac{1}{2 h}, \quad c_{\alpha, \alpha-1}^{*}=-\frac{1}{2 h} .
$$

The least squares slope is

$$
\bar{\sigma}_{\alpha}^{L S}=\frac{\bar{u}_{\alpha+1}-\bar{u}_{\alpha-1}}{2 h}
$$

The values of $\tilde{\psi}_{\alpha}, \tilde{\theta}_{\alpha}, \tilde{\psi}_{\alpha}$ in $(3.15)$ are

$$
\left\{\begin{array}{l}
\tilde{\psi}_{\alpha}=\frac{1}{(2 h)^{2}}\left(\bar{\sigma}_{\alpha+2}^{L S}+\bar{\sigma}_{\alpha-2}^{L S}-2 \bar{\sigma}_{\alpha}^{L S}\right) \\
\tilde{\theta}_{\alpha}=\frac{1}{2 h}\left(\bar{\sigma}_{\alpha+1}^{L S}-\bar{\sigma}_{\alpha-1}^{L S}\right) \\
\tilde{\sigma}_{\alpha}=\frac{53}{48} \bar{\sigma}_{\alpha}^{L S}-\frac{5}{96} \bar{\sigma}_{\alpha+2}^{L S}-\frac{5}{96} \bar{\sigma}_{\alpha-2}^{L S} .
\end{array}\right.
$$

In (4.6), $w_{\alpha}[V]\left(x_{\alpha, \alpha+1}\right)$ is given by:

$$
\begin{aligned}
w_{\alpha}[V]\left(x_{\alpha, \alpha+1}\right) & =w_{\alpha}[V]\left(x_{\alpha}+h / 2\right) \\
& =\bar{u}_{\alpha}+\frac{1}{2} h \tilde{\sigma}_{\alpha}+\frac{1}{12} h^{2} \tilde{\theta}_{\alpha}+\frac{1}{48} h^{3} \tilde{\psi}_{\alpha}
\end{aligned}
$$

The scheme (4.6) is therefore

$$
\begin{aligned}
\frac{d v_{\alpha}}{d t} & =-\frac{c}{h}\left[\left(v_{\alpha}(t)+\frac{h}{2} \tilde{\sigma}_{\alpha}(t)+\frac{h^{2}}{12} \tilde{\theta}_{\alpha}(t)+\frac{h^{3}}{48} \tilde{\psi}_{\alpha}(t)\right)\right. \\
& \left.-\left(v_{\alpha-1}(t)+\frac{h}{2} \tilde{\sigma}_{\alpha-1}(t)+\frac{h^{2}}{12} \tilde{\theta}_{\alpha-1}(t)+\frac{h^{3}}{48} \tilde{\psi}_{\alpha-1}(t)\right)\right] .
\end{aligned}
$$

We now give a dissipation and dispersion analysis of (4.15). Such an analysis is an important indication to interpret the numerical behaviour of the scheme. For each given wavenumber $k,-N / 1+1 \leq$ $k \leq N / 2$, we consider the periodic initial function $u_{0}^{k}(x)$ :

$$
u_{0}^{k}(x)=\exp (2 i k \pi x)
$$

The discrete initial data at $x_{\alpha}=\alpha h$ is

$$
u_{0, \alpha}^{k}=\exp \left(i \alpha \phi_{k}\right) \text { where } \phi_{k}=2 \pi k h \in(-\pi, \pi] .
$$

The solution of the system (4.7) is:

$$
u_{\alpha}^{k}(t)=\exp \left(2 i k \pi\left(x_{\alpha}-\hat{c}_{k} t\right)\right)
$$

where the numerical velocity $\hat{c}_{k}$, which depends on $k$, is decomposed into real and imaginary parts as

$$
\hat{c}_{k}=\hat{c}_{k, R}+i \hat{c}_{k, I}
$$

Definition 4.2. The dissipation and the dispersion functions are defined by

$$
\left\{\begin{array}{l}
\phi_{k} \in\left[0, \pi\left[\mapsto D_{1}\left(\phi_{k}\right)=\frac{\hat{c}_{k, I}}{c}, \quad(a)\right.\right. \\
\phi_{k} \in\left[0, \pi\left[\mapsto D_{2}\left(\phi_{k}\right)=\frac{\hat{c}_{k, R}}{c} \quad(b) .\right.\right.
\end{array}\right.
$$


- The dissipation function $D_{1}\left(\phi_{k}\right)$ is positive when the sinusoidal gridfunction function $z_{\alpha}^{k}=\exp \left(i \alpha \phi_{k}\right)$ is amplified for the wave number $k$. This corresponds to an unstable scheme. In the contrary, if $D_{1}\left(\phi_{k}\right) \leq 0$ for all $k$, then the scheme is stable.

- The dispersive function $D_{2}\left(\phi_{k}\right) \geq 1$ for a propagation at a velocity $\hat{c}_{k, R}>c$ and $D_{2}(\theta) \leq 1$ for a propagation at a velocity $\hat{c}_{k, R}<c$. In both cases, there is a dispersive behaviour of the scheme.

We proceed by expanding the normalized eigenvalue $s_{k}=c \lambda_{k} / h$ in powers of $h$ when $h \rightarrow 0$. This yields (the value $i \xi$ corresponds to the operator $\partial_{x}$ ):

$$
s_{k}=-c\left(i \xi+\frac{17}{384} i \xi^{5} h^{4}+\frac{229}{3840} \xi^{6} h^{5}\right)+O\left(h^{6}\right) .
$$

Otherwise stated, the modified equation [26] of the scheme (4.15) is

$$
\partial_{t} u+c \partial_{x} u=-c\left(\frac{17}{384} h^{4} \partial_{x}^{(5)} u(x, t)-\frac{229}{3840} h^{5} \partial_{x}^{(6)} u(x, t)\right)+O\left(h^{6}\right) .
$$

The relation (4.22) indicates fourth order accuracy with a leading dispersive error of order 4 . Furthermore the first dissipative term is of order 5 with a real part of $\lambda_{k}$ behaving as:

$$
\operatorname{Re}\left(\lambda_{k}\right) \sim-\frac{229}{3840} \phi_{k}^{6} \leq 0 .
$$

This suggests a stable behaviour. Actually the normalized full spectrum (see bottom panel of Fig. 4.2) confirms the stability of the scheme (4.15). The dissipation curve $\phi_{k} \in[0, \pi) \mapsto D_{1}\left(\phi_{k}\right)$ and the dispersion curve $\phi_{k} \in[0, \pi) \mapsto D_{2}\left(\phi_{k}\right)$ are shown on Fig. 4.2 (top panels).

Remark 4.3. Not all cubic reconstruction lead to a stable dynamical system (4.9). Consider for example the consistent approximations $\tilde{\sigma}_{\alpha}, \tilde{\theta}_{\alpha}$ and $\tilde{\psi}_{\alpha}$ to $u^{\prime}\left(x_{\alpha}\right), u^{\prime \prime}\left(x_{\alpha}\right)$ and $u^{\prime \prime \prime}\left(x_{\alpha}\right)$, defined by:

$$
\left\{\begin{array}{l}
\tilde{u}_{\alpha}+\frac{h^{2}}{24} \tilde{\theta}_{\alpha}=\bar{u}_{\alpha}, \\
\frac{1}{6} \tilde{\sigma}_{\alpha-1}+\frac{2}{3} \tilde{\sigma}_{\alpha}+\frac{1}{6} \tilde{\sigma}_{\alpha+1}=\delta_{x} \tilde{u}_{\alpha}, \\
\frac{1}{12} \tilde{\theta}_{\alpha-1}+\frac{10}{12} \tilde{\theta}_{\alpha}+\frac{1}{12} \tilde{\theta}_{\alpha+1}=2 \delta_{x}^{2} \tilde{u}_{\alpha}-\delta_{x} \tilde{\sigma}_{\alpha}, \\
\frac{1}{12} \tilde{\psi}_{\alpha-1}+\frac{10}{12} \tilde{\psi}_{\alpha}+\frac{1}{12} \tilde{\psi}_{\alpha+1}=\delta_{x}^{2} \tilde{\sigma}_{\alpha} .
\end{array}\right.
$$

An algebraic calculation, not shown here, demonstrates that the spectrum is located on the right of the imaginary axis and therefore that the scheme (4.7) with the reconstruction $w_{\alpha}[\overline{\mathfrak{u}}]$ in $(2.36)$ with $\tilde{\sigma}_{\alpha}, \tilde{\theta}_{\alpha}$ and $\tilde{\psi}_{\alpha}$ given in (4.24) is instable.

\section{One dimensional numerical results}

In this section, we display numerical results obtained with the scheme (4.2). First we give some results on the linear convection equation. In this case, (4.2) becomes (4.7). Then we show results for nonlinear gasdynamics test cases. 

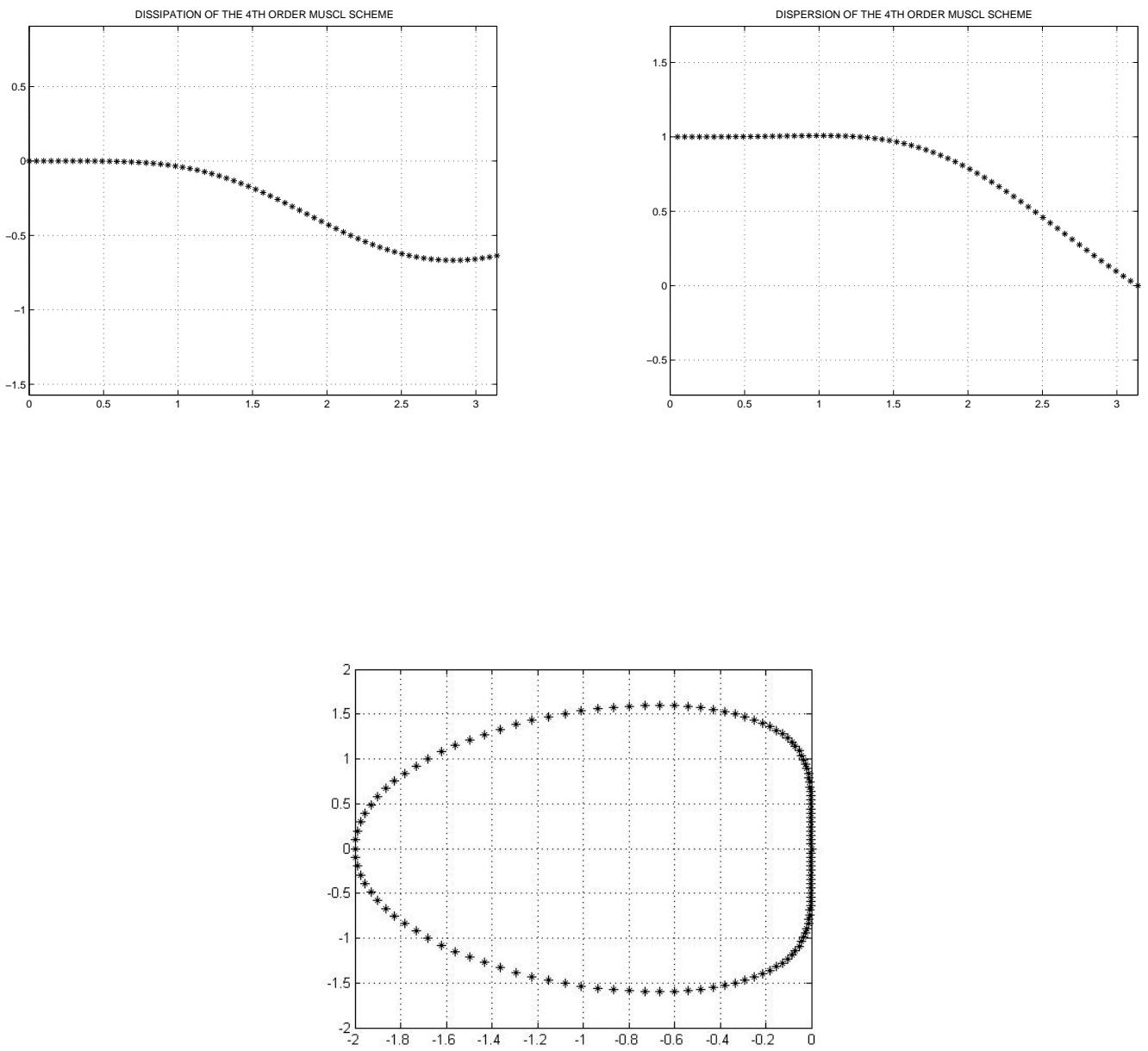

Figure 4.2: Top left panel: dissipation of the scheme (4.15). Top right panel: dispersion of the scheme (4.15). Bottom panel: spectrum of the matrix $J$ in (4.7). The spectrum is located in the left half-plane. This indicates stability. The order of accuracy is reflected by the tangency order at the origin along the vertical axis. 
In all the results the RK4 time stepping scheme is used. If $V^{n} \simeq V\left(t^{n}\right)$ is supposed given, then $V^{n+1}$ is computed by (in the case of (4.7)):

$$
\left\{\begin{array}{l}
k_{0}=\mathbf{J} V^{n} \\
k_{1}=\mathbf{J}\left(V^{n}+\frac{1}{2} \Delta t k_{0}\right), \\
k_{2}=\mathbf{J}\left(V^{n}+\frac{1}{2} \Delta t k_{1}\right), \\
k_{3}=\mathbf{J}\left(V^{n}+\Delta t k_{2}\right) \\
V^{n+1}=V^{n}+\Delta t\left(\frac{1}{6} k_{0}+\frac{1}{3} k_{1}+\frac{1}{3} k_{2}+\frac{1}{6} k_{3}\right) .
\end{array}\right.
$$

The scheme (5.1) is separately fourth order in space and time, and therefore it is globally fourth order (assuming a CFL condition for the time step.).

\subsection{Accuracy and convergence analysis for the linear advection equation}

In this section, we present an accuracy and convergence analysis for the linear convection equation

$$
\partial_{t} u+c \partial_{x} u=0, \quad c>0, \quad x \in[0,1],
$$

approximated by the scheme (4.7)-(5.1). Fourth order accuracy is expected. The grid refinement procedure is as follows. We start from a mildly irregular grid of $\Omega=[0,1]$, with $N$ cells $\mathcal{T}_{\alpha},\left|\mathcal{T}_{\alpha}\right|=\kappa_{\alpha} h$, $\sum_{\alpha} \kappa_{\alpha}=1$ defined by the law

$$
\kappa_{\alpha}=K\left(1+\frac{1}{2} \cos ^{2}\left(8 x_{\alpha} \pi\right)\right) .
$$

The constant $K$ is adjusted so that

$$
\sum_{\alpha=1}^{N} \kappa_{\alpha}=1 .
$$

After performing a first computation using this first grid, the grid is refined by randomly splitting each cell into two subcells with shapes corresponding to the two values

$$
\kappa_{2 \alpha-1}=\frac{K}{2} \kappa_{\alpha}\left(1-C r_{\alpha}\right), \quad \kappa_{2 \alpha}=\frac{K}{2} \kappa_{\alpha}\left(1+C r_{\alpha}\right),
$$

where $C$ is a constant monitoring the randomness of the refinement. The grid is refined five times according to (5.5). The result is a highly irregular grid. Fig. 5.1 reports the convergence of the scheme in maximum norm after 1,5 and 10 periods of propagation and using two different choices of the constant $C$. We observe very satisfactory error levels and convergence rates matching the expectation of fourth order accuracy. Note that there is little difference between the two refinement patterns.

\subsection{Shock tube problems}

In this section we display several results obtained for shock tube problems of gas dynamics. The one dimensional Euler system has the form (1.1)

$$
\partial_{t} q+\partial_{x} f=0
$$

with

$$
\left\{\begin{array}{l}
q=\left[\rho, \rho u, \rho\left(E+\frac{1}{2} u^{2}\right)\right]^{T} \\
f=\left[\rho u, \rho u^{2}+p,\left(\rho\left(E+\frac{1}{2} u^{2}\right)+p\right) u\right]^{T} .
\end{array}\right.
$$




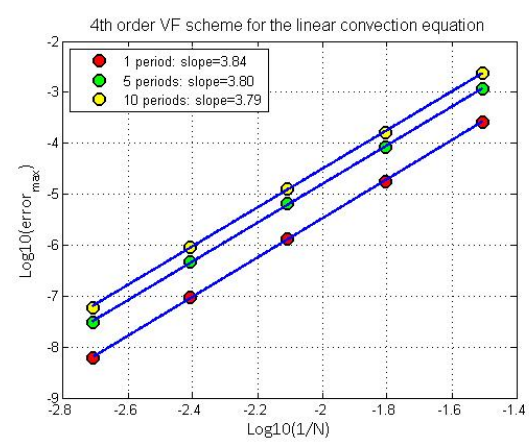

(a) Regular refinement with $C=0$ in (5.5)

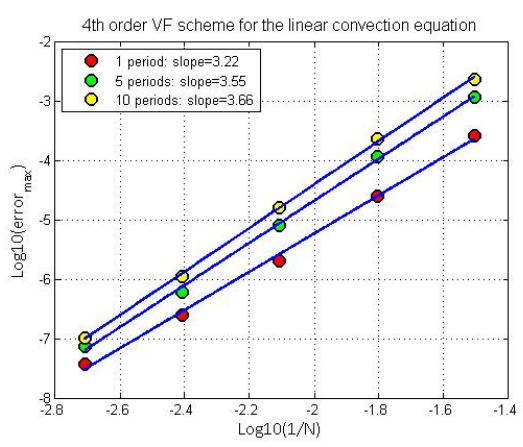

(b) Random refinement with $C=0.5$ in (5.5)

Figure 5.1: Fourth order finite volume scheme for the linear convection equation on a set of consistently refined irregular grids. The shape aspect of the cells is given by (5.3). Left panel: each cell is refined in two subcells. Right panel: random effect in the grid refinement, given in (5.5). In both case, an almost fourth order is observed after 1,5 and 10 periods.

The physical variables are $\hat{q}=[p, T, u]^{T}$. The thermodynamic data are given in dimensional form:

$$
\left\{\begin{array}{l}
r=285.7143 J \cdot \mathrm{kg}^{-1} \cdot K^{-1} \text { constant of perfect gas } \\
c_{v}=714.2857 J \cdot \mathrm{kg}^{-1} \cdot K^{-1} \\
p=r \rho T \text { and } E=c_{v} T \text { are the pressure and internal energy }
\end{array}\right.
$$

The semi discrete fourth order finite volume scheme for $t \mapsto V(t)=\left[v_{1}(t), v_{2}(t), \ldots, v_{N}(t)\right]^{T}$ is

$$
\frac{d v_{\alpha}(t)}{d t}=-\frac{1}{\left|\mathcal{T}_{\alpha}\right|}\left[f_{\alpha+1 / 2}(t)-f_{\alpha-1 / 2}(t)\right] .
$$

where $v_{\alpha}(t) \simeq q_{\alpha}(t)$, the average of $q(x, t)$ over $\mathcal{T}_{\alpha}$. The numerical flux function is chosen as the HLLC numerical flux [29]. But other numerical flux can be selected without significant influence on the result. The cubic reconstruction is performed on the physical variables using:

$$
w[V]_{\alpha}(x)=v_{\alpha}+\tilde{\sigma}_{\alpha}\left(x-x_{\alpha}\right)+\tilde{\theta}_{\alpha}\left(\frac{1}{2}\left(x-x_{\alpha}\right)^{2}-\frac{1}{24}\left|\mathcal{T}_{\alpha}\right|^{2}\right)+\frac{1}{6} \tilde{\psi}_{\alpha}\left(x-x_{\alpha}\right)^{3} .
$$

where the vector values $\tilde{\sigma}_{\alpha}, \tilde{\theta}_{\alpha}$ and $\tilde{\psi}_{\alpha}$, are obtained by the Algorithm 1 in Sec. 3. Again, the timestepping scheme is the RK4 scheme. We show the results obtained for two shock tube problems obtained with order the order two and four versions of the scheme, respectively. In both cases, we use the simple limiter (5.11) acting on the the physical variables. It is designed as follows. In each cell $\mathcal{T}_{\alpha}$, the reconstructed poynomial $w_{\alpha}[V]\left(x, t_{0}\right)$ is limited so that

$$
w_{\alpha}[V]\left(x_{\alpha \pm 1 / 2}\right) \in\left[\min _{\beta \in \mathcal{V}_{\alpha}} v_{\beta}, \max _{\beta \in \mathcal{V}_{\alpha}} v_{\beta}\right] .
$$

This is obtained by applying the same scalar coefficient $\lambda_{\alpha}$ to the three values $\tilde{\sigma}_{\alpha}, \tilde{\theta}_{\alpha}$ and $\tilde{\psi}_{\alpha}$ in $(2.36)$. In other words, the polynomial $w_{\alpha}[V](x)$ in $(5.10)$ is replaced by

$$
\tilde{w}_{\alpha}(x)=\bar{u}_{\alpha}+\lambda_{\alpha}\left(w_{\alpha}[V](x)-\bar{u}_{\alpha}\right)
$$

with $\lambda_{\alpha}>0$ such that (5.11) holds in the cell $\mathcal{T}_{\alpha}$. This limitation is part of the semi-discrete approximation. Therefore it is applied at each substep of the time scheme (5.1). Since this paper is focused on the 
reconstruction in Algorithm 1, we do not elaborate further on the limiter design and we limit ourselves to the limiter (5.11). Note that this limiter does not a priori prevent new extrema to appear. It is used for the sole purpose to limit nonlinear oscillations. Note also that in such shock tube problems, the physical time of simulation is too short to observe significant differences between the second order and the fourth order schemes. Future work will be devoted to a sharper comparison with a larger physical time of observation. Here a regular grid is used and our purpose is only to show that the fourth order scheme (4.2) is sufficiently robust to handle these gas dynamics test cases. The CFL $=0.3$ is selected in order for the time approximation to not significantly modify the accuracy in space.

\subsubsection{Sod test case}

In all our tests, the dimensions of the tube are:

$$
\left\{\begin{array}{l}
x_{0}=0.5 \mathrm{~m} \text { location of the initial discontinuity } \\
x_{l}=5.0 \mathrm{~m} \text { lenght of the half of the tube. }
\end{array}\right.
$$

For the Sod test, we use the initial left and right states in the unit system of (5.8):

$$
\left\{\begin{array} { l } 
{ \rho _ { l } = 3 . 5 \mathrm { kg } \cdot \mathrm { m } ^ { - 3 } } \\
{ u _ { l } = 0 . 0 \mathrm { m } \cdot \mathrm { s } ^ { - 1 } } \\
{ p _ { l } = 1 0 ^ { 6 } \mathrm { Pa } }
\end{array} \quad \left\{\begin{array}{l}
\rho_{r}=0.4375 \mathrm{~kg} \cdot \mathrm{m}^{-3} \\
u_{r}=0.0 \mathrm{~m} \cdot \mathrm{s}^{-1} \\
p_{r}=10^{5} \mathrm{~Pa}
\end{array}\right.\right.
$$

This corresponds to left and right temperature values of $T_{l}=1000 \mathrm{~K}$ and $T_{r}=800 \mathrm{~K}$, respectively. The observation time is $t=0.003 \mathrm{~s}$. The results are plotted against the exact solution. The contact discontinuity is slightly better with the scheme of order 4 (right column of Fig. 5.2) than with the one of order 2 (left column of Fig. 5.2). As already mentioned, due to the short observation time, there is no significant difference between the second order and the fourth order versions of the scheme. Observe that the limiter (5.11) (applied to the physical quantities $\hat{q}=[p, T, u]^{T}$ ) is sufficient to ensure a good behaviour of the scheme near discontinuities.

\subsubsection{Lax test case}

The Lax test case initial conditions are given using the physical system of units (5.8):

$$
\left\{\begin{array} { l } 
{ \rho _ { l } = 1 . 0 3 8 5 6 9 \mathrm { kg } \cdot \mathrm { m } ^ { - 3 } } \\
{ u _ { l } = 1 9 1 . 5 1 1 \mathrm { m } \cdot \mathrm { s } ^ { - 1 } } \\
{ p _ { l } = 6 . 1 7 8 0 0 1 0 ^ { 5 } \mathrm { Pa } }
\end{array} \quad \left\{\begin{array}{l}
\rho_{r}=1.16667 \mathrm{~kg} \cdot \mathrm{m}^{-3} \\
u_{r}=0.0 \mathrm{~m} \cdot \mathrm{s}^{-1} \\
p_{r}=10^{5} \mathrm{~Pa}
\end{array}\right.\right.
$$

This corresponds to left and right temperature values of $T_{l}=2082 \mathrm{~K}$ and $T_{r}=300 \mathrm{~K}$, respectively. The observation time is $t=0.00575 \mathrm{~s}$. Again there is no significant difference between the versions of order 2 (Fig. 5.3) and 4 (right column of Fig. 5.3). We only observe a better resolution of the contact discontinuity with the fourth order scheme. In this case, the limiter (5.11) is not sufficient to limit the overshoot near the contact discontinuity. We oberve this behaviour in the case of a temperature transition of the form $T_{\text {before }}<T_{\text {after }}$. Note that no overshoot was observed in the opposite case ( $\left.T_{\text {after }}<T_{\text {before }}\right)$. Observe nonetheless that the fourth order scheme is stable. 

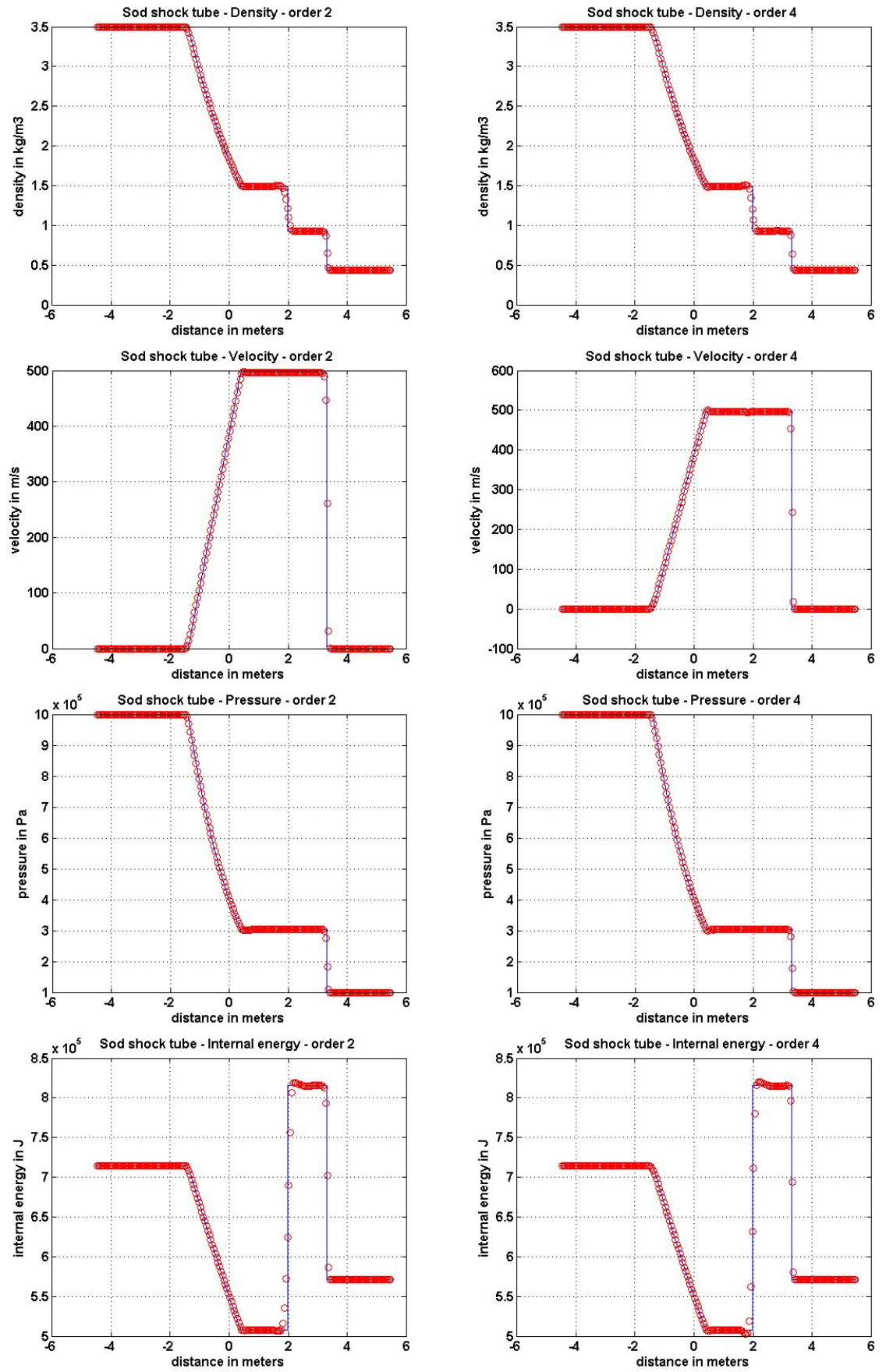

Figure 5.2: Sod shock tube. Finite volume scheme with a regular grid of 200 cells. Left column: standard second order reconstruction. Right column: finite volume scheme with fourth order reconstruction (5.10). In both cases, the slope limiter is (5.11) and $\mathrm{CFL}=0.3$. The exact solution is represented with the continuous line. 

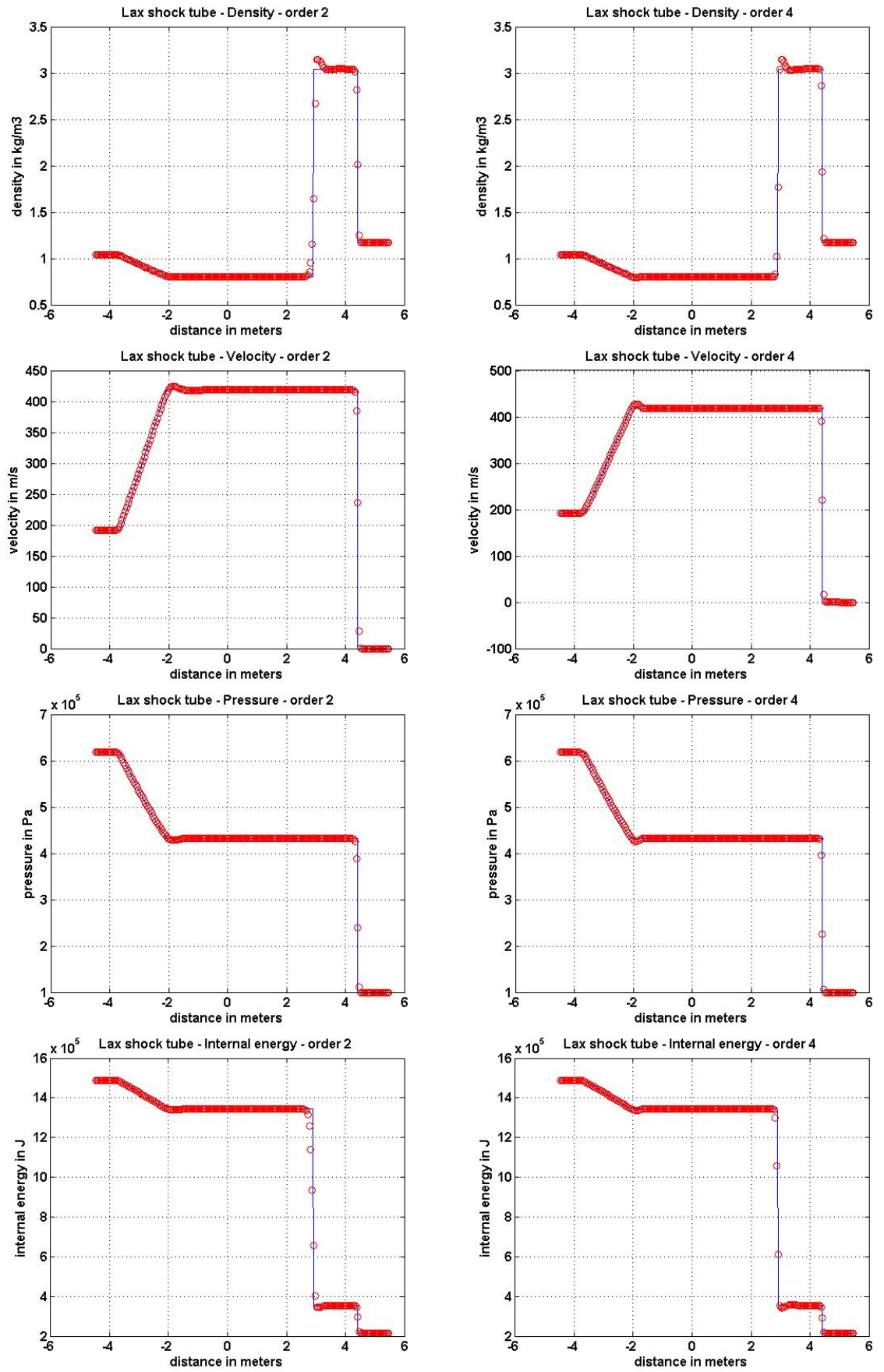

Figure 5.3: Lax shock tube. Finite volume scheme with a regular grid of 200 cells. Left column: standard second order reconstruction. Right column: finite volume scheme with fourth order reconstruction (5.10). In both cases, the slope limiter is (5.11). The CFL is CFL $=0.3$. The exact solution is represented with the continuous line. 

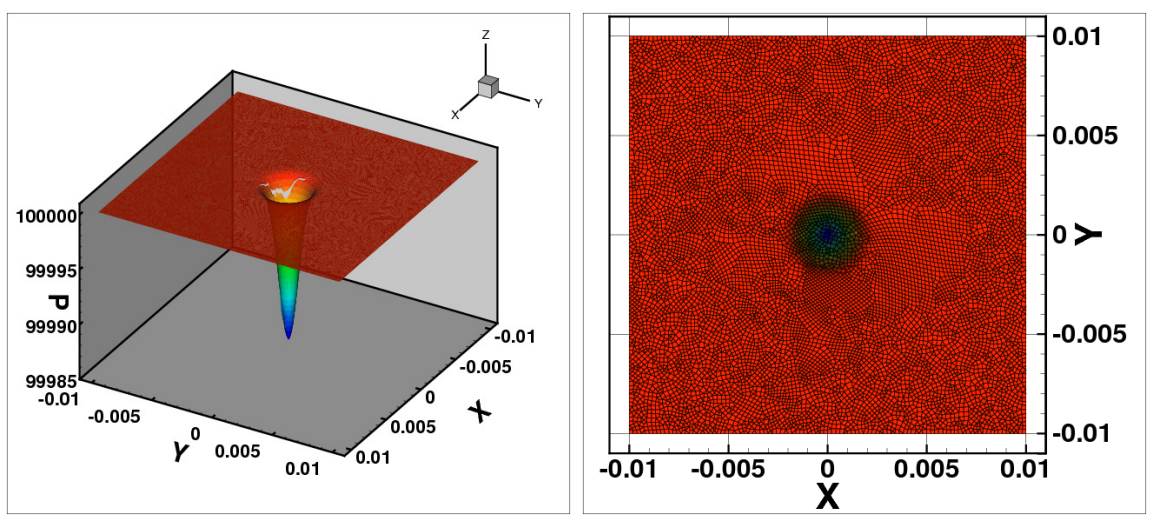

Figure 6.1: Convection of the isothermal vortex (6.1) - Left panel: initial condition. Right panel: irregular grid.

\section{Multidimensional numerical results}

The two test cases displayed hereafter are related to propagation problems in gas dynamics. Both aim to illustrate the efficiency of the cubic reconstruction procedure on an irregular grid when compared to lower order reconstructions.

\subsection{Vortex transport by a uniform flow}

The first test case consists of an isothermal Gaussian vortex moving accross a periodic box with constant velocity. This vortex is an analytical solution of the two dimensional Euler equations. The dimensional characteristics of the vortex are as follows:

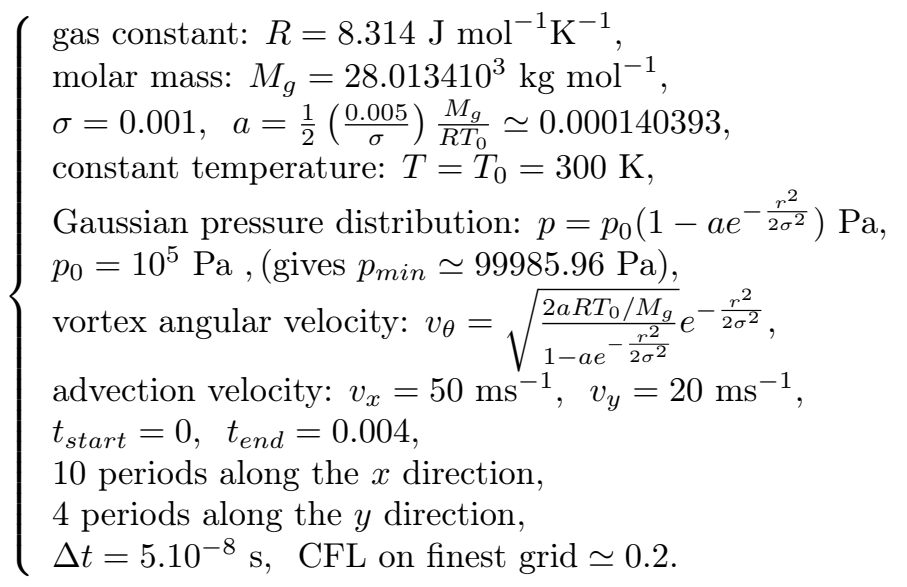

A piecewise cubic reconstruction of the form (2.28) is used combined with the Roe numerical flux. There is no limitation needed for this case. At final time Fig. 6.2 reports the shape of the vortex when using the irregular grid in the right panel of Fig. 6.1. The vortex spans over approximately 15 cells. As can be observed, after several periods of propagation, there is a drastic difference between the piecewise affine reconstruction and the piecewise cubic reconstruction. For comparison the result using a $200 \times 200$ Cartesian grid is also reported. Comparing Fig. 6.2 and 6.3, it turns out that the result obtained with the fourth order scheme on the irregular grid is more accurate than the result obtained using the Cartesian grid with the second order scheme. 


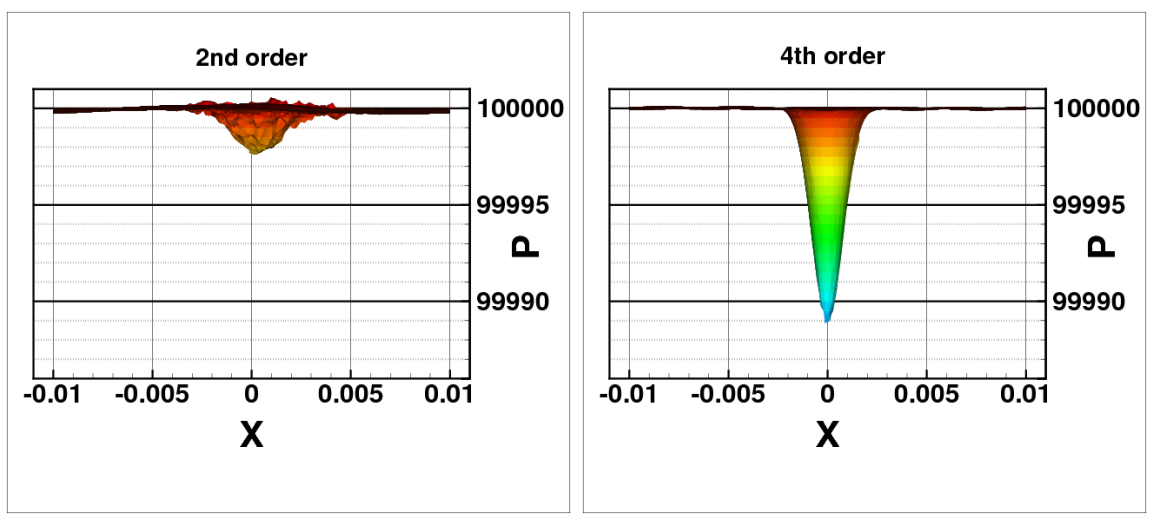

Figure 6.2: Convection of the isothermal vortex (6.1) with the unstructured grid in Fig. 6.1 at final time - Left panel: piecewise linear reconstruction (second order FV scheme) - Right panel: piecewise cubic reconstruction , (fourth order FV scheme).
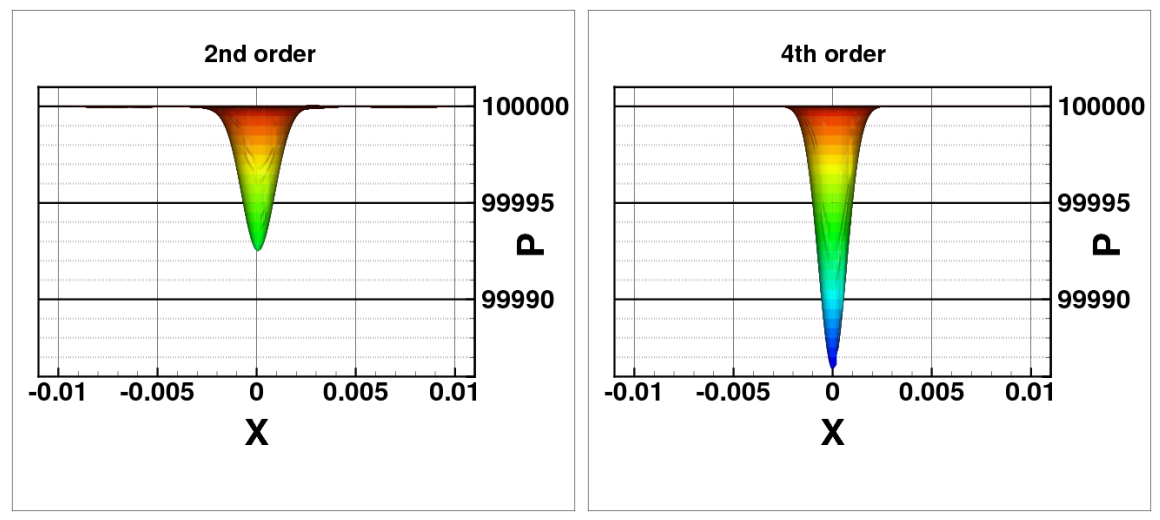

Figure 6.3: Convection of the isothermal vortex (6.1) with a Cartesian fine grid at final time - Left panel: piecewise linear reconstruction (second order FV scheme) - Right panel: piecewise cubic reconstruction, (fourth order FV scheme). 


\subsection{Acoustic Wave in a 3D Channel}

This test case concerns a propagation problem typically occuring in aeroacoustics. Aeroacoustics computations are usually performed by associating two kinds of codes:

- an unsteady CFD code for LES (Large Eddy Simulation). This first code calculates acoustic sources generated by the turbulent flow and performs the numerical propagation in the acoustic near field region,

- an acoustic code to reconstruct the far field from unsteady CFD results recorded at CFD-acoustic interfaces (e.g. [25]).

It is thus very important to assert the efficiency of the CFD code for accurately propagating acoustic waves in the near field up to the CFD/acoustic interface without excessive damping or phase errors. Complex geometries are to be considered frequently and unstructured meshes are most of the time mandatory. In general, when $2^{\text {nd }}$ order CFD schemes are used, fine meshes are required in the near field region to compensate the limited frequency bandwidth of these schemes. In $[6,20]$ numerical results are shown with a $2^{\text {nd }}$ order scheme for aeroacoustics problems. It is believed that higher order accurate schemes will demonstrate higher bandwidth thus allowing less refined meshes for a fixed precision. A popular measure of the bandwidth of a scheme is simply expressed as the minimum number of grid points (or cells) per wavelength (denoted by $p p w$ ). This is required to properly propagate a given wave pattern over a significant length [5]. When using a $2^{\text {nd }}$ order schemes, a $p p w$ of the order of 20-25 is often mentioned while with recent high order schemes, the $p p w$ parameter is likely of the order 4-6.

\subsubsection{Test case outline}

Our test case consists of an acoustic wave propagating in a uniform flow. The geometry consists of a square duct with axis $O x$ and uniform cross section of $0.2 \times 0.2 \mathrm{~m}$. The length is $\mathrm{L}=10 \mathrm{~m}$. The duct is filled with a uniform flow of air at $V x=1 \mathrm{~m} \cdot \mathrm{s}^{-1}$ at ambient conditions: $101325 \mathrm{~Pa}$ and $300 \mathrm{~K}$. Under these conditions the sound velocity is $c=347 \mathrm{~m} \cdot \mathrm{s}^{-1}$. Boundary conditions are set to:

- left edge ( $x=0)$ : subsonic inlet conditions set at $V x=1 \mathrm{~m} \cdot \mathrm{s}^{-1}, T=300 \mathrm{~K}$.

- right edge $(x=L)$ : subsonic outlet conditions set at $\mathrm{P}=101325 \mathrm{~Pa}$.

- lateral surfaces : slip boundary conditions.

We have used a uniform mesh of isotropic tetrahedra of size $a=20 \mathrm{~mm}$. At the right edge, four layers of prisms of uniform size of $16 \mathrm{~mm}$ were added. This part of the grid intends to allow for a planar acoustic wave generation as described below. Overall, the grid consists of 288876 cells and 600053 faces, (see fig. $6.4)$.

The simulation is initiated by a sinusoidal perturbation at the right end of the duct: a time harmonic, isothermal $(300 \mathrm{~K})$, distributed mass source enters the first layer of prismatic cells on the right of the computational domain. It is expressed as:

$$
\dot{m}=A \sin (2 \pi f t) .
$$

In (6.2) the amplitude $A$ is set so as to generate a low amplitude acoustic wave (smaller than $20 \mathrm{~Pa}$ of physical amplitude) such that non linear effects can be ignored. This source generates a plane acoustic wave propagating upstream, from the right to the left with a phase velocity $V_{\varphi}=c-V_{x}=346 \mathrm{~m} \cdot \mathrm{s}^{-1}$. The corresponding wavelength is thus:

$$
\lambda=\frac{V_{\varphi}}{f} .
$$




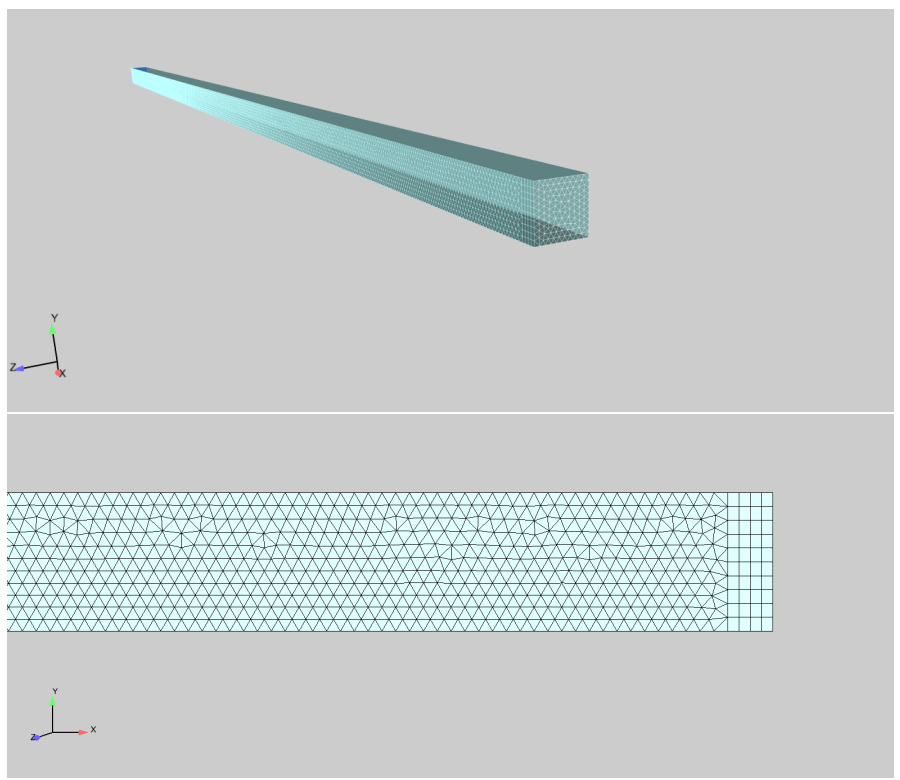

Figure 6.4: Test Case 1: Acoustic wave in a channel - Duct overview and grid details at the right end

The physical elapsed time of each run is $20 \mathrm{~ms}$. This corresponds to a propagation length of $6.9 \mathrm{~m}$ in the counter flow direction $(x<0)$. The inlet boundary is therefore not impacted, thus alleviating any acoustic reflection issues at the left end boundary. A total of 7578 time steps are performed at $\mathrm{CFL}=0.4$. By using a series of values for the source frequency $f$, the resolution of the acoustic wave for a given grid, as represented by the parameter ppw (number of grid points per wavelength) is estimated by

$$
p p w=\frac{\lambda}{a} .
$$

Runs were performed for a series of 4 values of the frequency $f$, which corresponds to a ppw number varying from 6 (coarse mesh) to 59 (extra fine mesh). Table 1 summarizes the results. When $2^{\text {nd }}$ order

\begin{tabular}{lcccc}
\hline Run & R1 & R2 & R3 & R4 \\
\hline $\mathrm{f}(\mathrm{Hz})$ & 300 & 700 & 1500 & 3000 \\
$\lambda(\mathrm{m})$ & 1.1533 & 0.4943 & 0.2307 & 0.1153 \\
$\mathrm{ppw}=\lambda / \mathrm{a}$ & 59 & 25 & 12 & 6 \\
\hline
\end{tabular}

Table 1: Test Case 1: Acoustic wave in a channel - Values of the wave frequency

CFD schemes are used in aeroacoustic, a value of 20-25 ppw is typically used. This corresponds to a specific grid step size, according to the expected frequency range of the acoustic sources. This value was selected for the runs of the R2 series. Then for each run conditions, computations were performed for schemes of various orders, as described in Table 2 , and the wave quality was observed and compared at different numerical transducers located along the duct axis. 

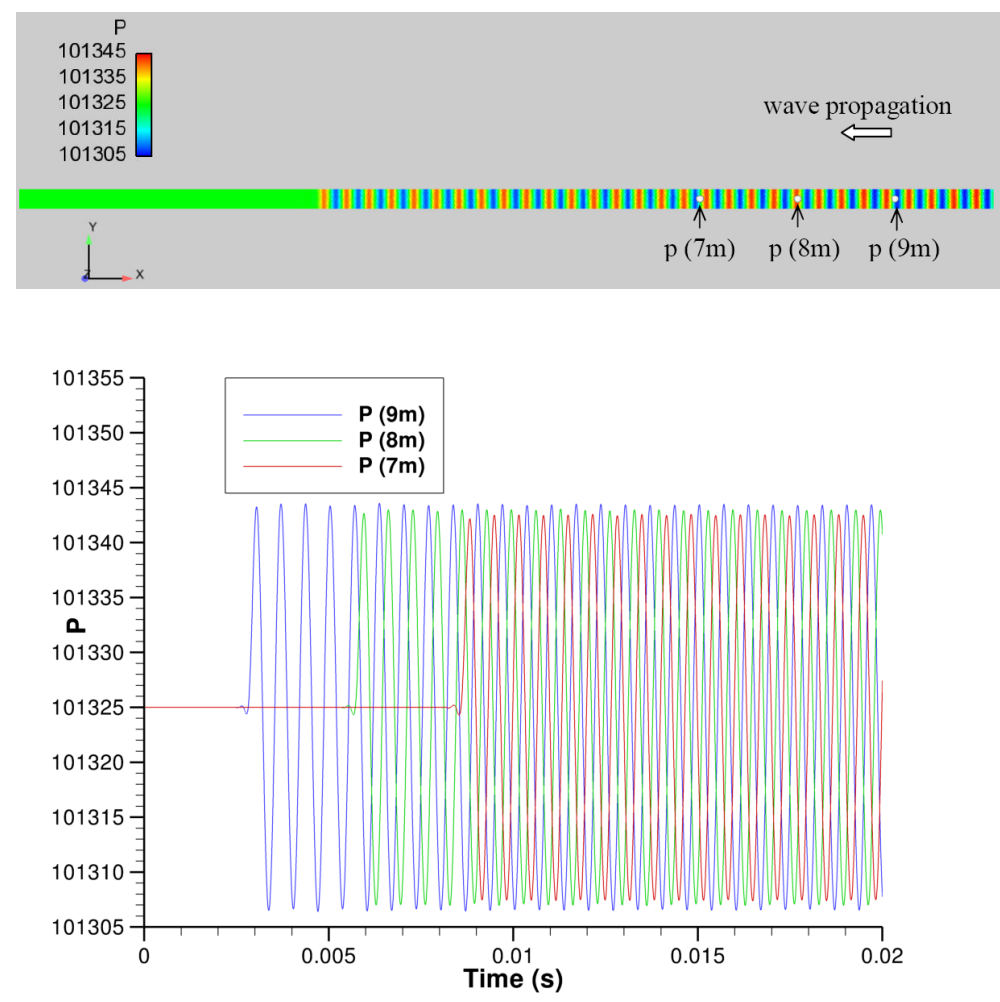

Figure 6.5: Test case 1: Acoustic wave in a channel - Top: illustration of wave propagation - Bottom: pressure time histories at numerical transducers location. Numerical case R3 and scheme O4-RK4 (see Tables 1 and 2)

\subsubsection{Amplitude and phase error analysis}

The analysis of the numerical results was performed using three numerical transducers located on the duct axis at $7 \mathrm{~m}, 8 \mathrm{~m}$ and $9 \mathrm{~m}$, respectively, see Fig. 6.5 top. For example we show on Fig. 6.5 (bottom) the pressure time history for a specific run.

The quality of the schemes was estimated from the wave amplitude damping along the propagation direction. The phase error was measured with respect to an ideal linear acoustic wave propagation solution of the form

$$
P\left(x, t_{i}\right)=\operatorname{Re}\left[P_{0} e^{i\left(\omega t-k r+\varphi_{0}\right)}\right]
$$

with $\omega=2 \pi f \in \mathbb{R}$ and $k=k^{r}+i k^{i} \in \mathbb{C}$. The calculated time series is assumed to have the form

$$
F\left(x, t_{i}\right)=A_{0}(x) \sin \left(2 \pi f(x) t_{i}+\varphi(x)\right) e^{-\alpha(x) t_{i}} .
$$

where the four parameter functions $A_{0}(x), f(x), \varphi(x)$ and $\alpha(x)$ have to be estimated. Identifying these four functions is obtained by a fitting approximation technique using exponential (in time) sums. This kind of fitting is thoroughly presented e.g. in [32]. Here we assume that our numerical data can be represented by the series

$$
F\left(x, t_{i}\right)=\sum_{n=1}^{N} a_{n} e^{i \sigma_{n} t_{i}} \text { with }\left(a_{n}, \sigma_{n}\right) \in \mathbb{Z}^{2}
$$

The function $F$ represents a real monochromatic wave, so that we limit the sum to two terms $(N=2$ in (6.7)). By identification the functions $A_{0}(x), f(x), \varphi(x)$ and $\alpha(x)$ are constants and they are expressed 
in terms the values $a_{1}, a_{2}, \sigma_{1}$ and $\sigma_{2}$ as

$$
\begin{aligned}
A_{0} & =\sqrt{4 a_{1} a_{2}}, & 2 \pi f & =\frac{\sigma_{1}-\sigma_{2}}{2}, \\
\varphi & =\frac{1}{2 i} \log \left(\frac{-a_{1}}{a_{2}}\right), & \alpha & =\frac{\sigma_{1}+\sigma_{2}}{2 i} .
\end{aligned}
$$

An iterative least squares procedure is then used to identify these four constants. Turning back to the damping and dispersion evaluation, we use the now estimated functions $A_{0}(x)$ and $\varphi(x)$ as a basis of our measurement ${ }^{4}$. The numerical wave damping for one single wavelength is estimated by

$$
\begin{aligned}
\frac{A_{0}\left(x_{2}\right)}{A_{0}\left(x_{1}\right)} & =e^{k^{i}\left(x_{2}-x_{1}\right)}=e^{\lambda k^{i}\left(\frac{x_{2}-x_{1}}{\lambda}\right)}, \\
e^{\lambda k^{i}} & =e^{\left(\frac{\lambda}{x_{2}-x_{1}}\right) \log \left(\frac{A_{0}\left(x_{2}\right)}{A_{0}\left(x_{1}\right)}\right)},
\end{aligned}
$$

where $e^{\lambda k^{i}}$ represents the wave damping per wavelength traveled. Similarly, the numerical dispersion can be represented by the phase difference between two transducer locations as

$$
\begin{aligned}
\Delta \varphi_{21} & =\varphi\left(x_{2}\right)-\varphi\left(x_{1}\right), \\
\left(\Delta \varphi_{21}\right)_{\mathrm{th}} & =-k^{r}\left(x_{2}-x_{1}\right),
\end{aligned}
$$

where $k^{r}=\frac{2 \pi f}{V_{\varphi}}$. The relative error on the phase velocity error can be finally expressed as

$$
\frac{\partial V_{\varphi}}{V_{\varphi}}=\frac{\partial\left(\Delta \varphi_{21}\right)}{\frac{2 \pi\left(x_{2}-x_{1}\right)}{\lambda}}
$$

\subsubsection{Numerical Results}

Results using reconstruction of order 2, 3 and 4 (denoted by $O 2, O 3$, and $O 4$ ) are reported. This corresponds to reconstruction polynomials of order 1,2 and 3 respectively. The comparison analysis of Sec 6.2.2 was carried out with the results obtained from different runs summarized in Table 2.

\begin{tabular}{lcccc}
\hline Run & R1 & R2 & R3 & R4 \\
\hline O2 - RK2 & $\mathrm{x}$ & $\mathrm{x}$ & $\mathrm{x}$ & $\mathrm{x}$ \\
$\mathrm{O} 3-\mathrm{RK} 3$ & & $\mathrm{x}$ & $\mathrm{x}$ & $\mathrm{x}$ \\
$\mathrm{O} 4-\mathrm{RK} 4$ & & $\mathrm{x}$ & $\mathrm{x}$ & $\mathrm{x}$ \\
$\mathrm{O} 4-\mathrm{RK} 4, \mathrm{CFL}=0.8$ & & & $\mathrm{x}$ & \\
\hline
\end{tabular}

Table 2: Test Case 6.2: acoustic wave in a channel - Computation matrix

Tables 3 and 4, and Figs. 6.6 and 6.7 show that the scheme of order 4 provides a drastic improvement in the ability of the code to propagate acoustic waves. Even on a coarse grid (up to 6 points per wavelength) a very small damping and phase error was observed. Regarding a detailed comparison, Fig. 6.6 shows a similar damping between $2^{\text {nd }}$ order and the $3^{\text {rd }}$ order scheme whereas Fig. 6.7 shows that the $3^{\text {rd }}$ order and the $4^{\text {th }}$ order schemes have a similar phase error. This is easily interpreted in terms of the modified equation (see (4.22) and [26]) associated with the linear transport equation. A $2^{\text {nd }}$ and a $3^{\text {rd }}$ order scheme have a first dissipative error $h^{3} \partial_{x}^{(4)} u$, whereas a $3^{\text {rd }}$ and $4^{\text {th }}$ order schemes have a

\footnotetext{
${ }^{4}$ Note that the two other functions $f(x)$ and $\alpha(x)$ are used only to check the accuracy of the exponential fitting.
} 


\begin{tabular}{lcccc}
\hline Run (ppw) & O2-RK2 & O3-RK3 & O4-RK4 & O4-RK4(CFL =0.8) \\
\hline R1 (59) & 0.9995 & & & \\
R2 (25) & 0.9961 & 0.9965 & 0.9990 & \\
R3 (12) & 0.9843 & 0.9866 & 0.9942 & 0.9939 \\
R4 (6) & 0.9188 & 0.9276 & 0.9754 & \\
\hline
\end{tabular}

Table 3: Test Case 6.2: acoustic wave in a channel - Summary of results for wave damping per wavelength

\begin{tabular}{lrrrc}
\hline Run (ppw) & O2-RK2 & O3-RK3 & O4-RK4 & O4-RK4(CFL = 0.8) \\
\hline R1 (59) & $-0.0119 \%$ & & & \\
R2 (25) & $-0.0695 \%$ & $-0.0054 \%$ & $0.0000 \%$ & \\
R3 (12) & $-0.3046 \%$ & $-0.0183 \%$ & $-0.0256 \%$ & $-0.0287 \%$ \\
R4 (6) & $-1.0516 \%$ & $-0.0466 \%$ & $-0.0744 \%$ & \\
\hline
\end{tabular}

Table 4: Test Case 6.2: acoustic wave in a channel - Summary of results for the relative phase velocity error (6.11)

\begin{tabular}{lcccc}
\hline $\mathrm{a}$ & O2-RK2 & O3-RK3 & O4-RK4 & O4-RK4 $(\mathrm{CFL}=0.8)$ \\
\hline Intrinsic CPU ratio & 1 & 4.7 & 12.0 & 12.0 \\
Run CPU ratio & 1 & 4.7 & 12.0 & 6.0 \\
\hline
\end{tabular}

Table 5: Test Case 6.2: acoustic wave in a channel - Relative CPU cost

first dispersive error of the form $h^{4} \partial_{x}^{(5)} u$. Overall the $4^{\text {th }}$ order scheme detailed in Sections 3 and 4 outperforms the two other schemes.

Table 5 reports numerical values regarding computational cost. The computational complexity of the $3^{\text {rd }}$ and $4^{\text {th }}$ schemes was numerically measured against the $2^{\text {nd }}$ order scheme. Two values are reported. First the intrinsic CPU ratio, which is a numerical evaluation of the cost of one single time iteration. Second the CPU ratio for the full simulation. The latter also depends on the stability properties of each scheme. As an example, the RK4 (explicit) time stepping scheme allows a stability limit of CFL $=0.8$ with the $4^{\text {th }}$ order scheme instead of $\mathrm{CFL}=0.4$ with the second order scheme. The overall CPU ratio is therefore reduced to a factor, as reported in Table 5. The change from CFL $=0.4$ to CFL $=0.8$ did not bear any loss in terms of scheme accuracy, as shown in Tables 3 and 4 and in Fig. 6.6 and 6.7.

\section{Conclusion}

This paper describes the Coupled Least Squares reconstruction procedure to be used in finite volume methods. This piecewide cubic reconstruction belongs to the $k$ - exact reconstruction of [4]. We have investigated the design and implementation of this reconstruction on irregular grids. We have obtained a high order finite volume scheme which can be extended to complex physical models. The resulting scheme exhibits good stability properties.

As explained in the introduction, our main interest is to keep the paradigm of one unknown per cell, avoiding to introduce additional degrees of freedom in the cells. Furthermore, our reconstruction procedure is fully centered. In particular no solution dependent reconstruction stencils are used. Since the algorithm needs data located in a compact neighborhood, the reconstruction can be efficiently implemented regarding parallellism. This is clearly of crucial importance for practical applications. This property will be further analyzed elsewhere in physically realistic situations. 


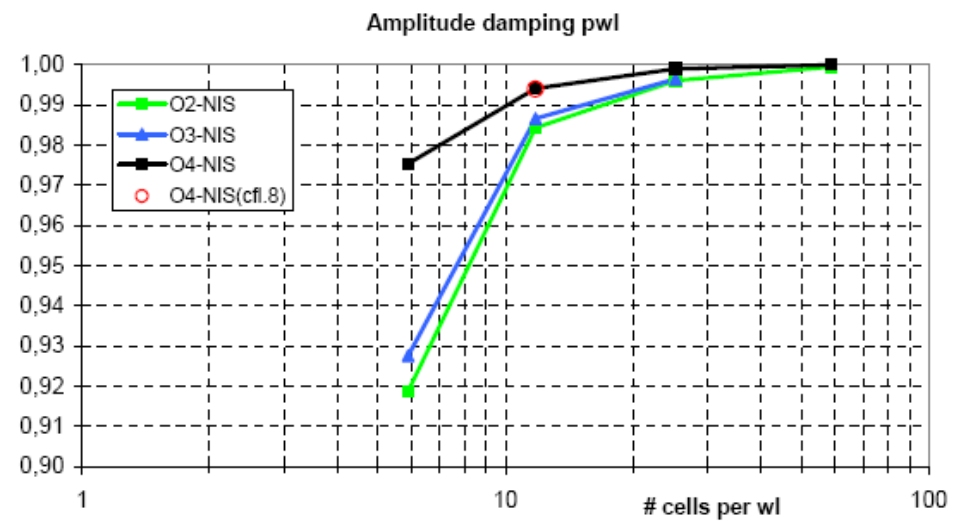

Figure 6.6: Test Case 6.2: acoustic wave in a channel - Wave damping per wavelength

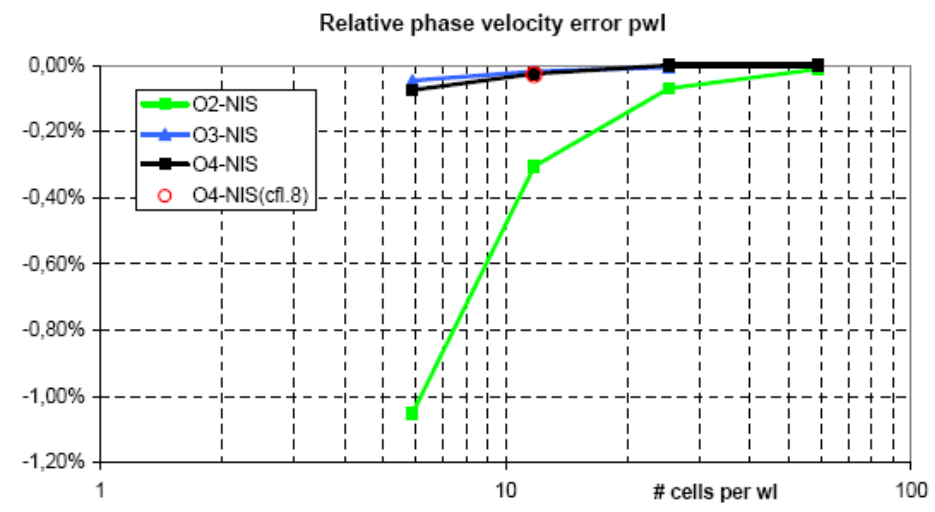

Figure 6.7: Test Case 6.2: acoustic wave in a channel - Relative phase velocity error

\section{Appendix: Tensor notation}

The tensor formalism permits to handle the algebra for polynomial reconstruction in multidimension. Refer on this topic to the recent monograph [11]. Here we review the definitions needed in Section 2.1. We follow $[12,13]$. A tensor a of order $k$ is a linear application

$$
\left(\mathbf{x}_{1}, \ldots, \mathbf{x}_{k}\right) \in\left(\mathbb{R}^{d}\right)^{k} \mapsto \mathbf{a}^{(k)}\left(\mathbf{x}_{1}, \ldots, \mathbf{x}_{k}\right) .
$$

The components of $\mathbf{a}^{(k)}$ are

$$
a_{i_{1} \ldots i_{k}}=\mathbf{a}^{(k)}\left(\mathbf{e}_{i_{1}}, \ldots \mathbf{e}_{i_{k}}\right) .
$$

The tensor $\mathbf{a}^{(k)}$ is symmetric if for all permutation $\sigma$ of $k$ elements,

$$
a_{\sigma\left(i_{1}\right) \ldots \sigma\left(i_{k}\right)}=a_{i_{1} \ldots i_{k}} .
$$

In Section 2.1 is used the fact that the derivative $D^{(k)} u(\mathbf{x})$ is a symmetric tensor of order $k$ with components

$$
D^{(k)} u_{i_{1} \ldots i_{k}}=\frac{\partial u(\mathbf{x})}{\partial x_{i_{1}} \ldots \partial x_{i_{k}}}, \quad 1 \leq i_{1} \ldots i_{k} \leq d
$$


Finally for $0 \leq m \leq k$, the product of the tensors $\mathbf{a}^{(k)}$ and $\mathbf{b}^{(m)}$ is the tensor of order $k-m$ denoted by $\mathbf{a}^{(k)} \cdot \mathbf{b}^{(m)}$ with components

$$
\left(\mathbf{a}^{(k)} \cdot \mathbf{b}^{(m)}\right)_{i_{1} \ldots i_{k-m}}=\sum_{j_{1}=1}^{d} \cdots \sum_{j_{m}=1}^{d} a_{i_{1} \ldots i_{k-m} j_{1} \ldots j_{m}} b_{j_{1} \ldots j_{m}} .
$$

In the particular case $m=k$, the product of $\mathbf{a}^{k}$ and $\mathbf{b}^{k}$ is called the contraction and is the scalar denoted by

$$
\mathbf{a}^{(k)} \bullet \mathbf{b}^{(k)}=\sum_{j_{1}=1}^{d} \cdots \sum_{j_{k}=1}^{d} a_{j_{1} \ldots j_{k}} b_{j_{1} \ldots j_{k}} .
$$

Finally the tensor product of $\mathbf{a}^{(k)}$ and $\mathbf{b}^{(m)}$ is the tensor $\mathbf{c}^{(k+m)}=\mathbf{a}^{(k)} \otimes \mathbf{b}^{(m)}$ with components

$$
c_{i_{1}, \ldots i_{k+m}}=a_{i_{1} \ldots i_{k}} b_{i_{k+1} \ldots i_{k+m}} .
$$

In particular, for $\mathbf{x} \in \mathbb{R}^{d}$ a given vector, the tensor $\mathbf{x}^{\otimes k}$ is defined by

$$
x_{i_{1} \ldots i_{k}}^{\otimes k}=x_{i_{1}} \ldots x_{i_{k}} .
$$

In our context, specific tensor notation is as follows. Cell indices are $\alpha$ and $\beta \in \mathbb{V}_{\alpha}$.

$$
\left\{\begin{array}{l}
\mathbf{x}_{\alpha}=\left(x_{\alpha, 1}, \ldots, x_{\alpha, d}\right)^{T} \in \mathbb{R}^{d} \text { barycenter of } \mathcal{T}_{\alpha}, \\
\mathbf{h}_{\alpha \beta}=\mathbf{x}_{\beta}-\mathbf{x}_{\alpha}, \text { with norm } h_{\alpha \beta}=\left|\mathbf{h}_{\alpha \beta}\right|, \\
\mathbf{h}_{\alpha \beta}^{(k)}=\underbrace{\mathbf{h}_{\alpha \beta} \otimes \cdots \otimes \mathbf{h}_{\alpha \beta}}_{k \times}, \\
\mathbf{z}_{\alpha \beta}^{(k)}=\frac{1}{\left|\mathcal{T}_{\beta}\right|} \int_{\mathcal{T}_{\beta}}^{k \times}\left(\mathbf{x}-\mathbf{x}_{\alpha}\right)^{\otimes k} d \mathbf{x}, \\
\mathbf{x}_{\alpha}^{(k)}=\mathbf{z}_{\alpha \alpha}^{(k)}, \\
\left|\mathcal{T}_{\alpha}\right|=\int_{\mathcal{T}_{\alpha}} d \mathbf{x}=\mathbf{x}_{\alpha}^{(0)} .
\end{array}\right.
$$

In particular we have

$$
\mathbf{z}_{\alpha}^{(1)}-\mathbf{x}_{\alpha}^{(1)}=\mathbf{h}_{\alpha \beta}^{(1)}=\mathbf{h}_{\alpha \beta} .
$$

The components of the tensor $\mathbf{z}_{\alpha \beta}^{(k)}$ are $z_{\alpha \beta, i_{1} i_{2} \ldots i_{k}}^{(k)}$ defined for $1 \leq i_{1} \ldots i_{k} \leq d$ by

$$
z_{\alpha \beta, i_{1} i_{2} \ldots i_{k}}^{(k)}=\int_{\mathcal{T}_{\beta}}\left(x_{i_{1}}-x_{\alpha, i_{1}}\right)\left(x_{i_{2}}-x_{\alpha, i_{2}}\right) \ldots\left(x_{i_{k}}-x_{\alpha, i_{k}}\right) d \mathbf{x}
$$

Moreover the consistency of the reconstruction is estimated using the scale $h$ which is such that

$$
c h^{d} \leq\left|\mathcal{T}_{\alpha}\right| \leq C h^{d}, \quad c h \leq h_{\alpha \beta} \leq C h, \quad 1 \leq \alpha, \beta \leq N,
$$

for some constants $c$ and $C$. 


\section{Appendix: Spatial accuracy analysis}

Here we give some comments on the accuracy analysis of the approximation in space developed in this paper. Let $u(x, t)$ be solution of the advection equation $\partial_{t} u=\partial_{x} u$. Averaging over the cell $\mathcal{T}_{\alpha}$ gives (see Sec. 4.1):

$$
\frac{d \bar{u}_{\alpha}(t)}{d t}=\frac{1}{h_{\alpha}}\left[u\left(x_{\alpha+1 / 2}, t\right)-u\left(x_{\alpha-1 / 2}, t\right)\right], \quad \text { with } h_{\alpha}=\left|\mathcal{T}_{\alpha}\right| .
$$

The approximation $t \mapsto v_{\alpha}(t)$ of $\bar{u}_{\alpha}(t)$ is solution of:

$$
\frac{d v_{\alpha}(t)}{d t}=\frac{1}{h_{\alpha}}\left[w_{\alpha}[V(t)]\left(x_{\alpha+1 / 2}^{-}\right)-w_{\alpha}[V(t)]\left(x_{\alpha-1 / 2}^{-}\right)\right] .
$$

where $V(t)=\left[v_{1}(t), \ldots, v_{N}(t)\right]$ and $w_{\alpha}[V](x)$ is the cubic polynomial (2.36). Let $u(x)$ be any regular function and $U=\left[\bar{u}_{1}, \ldots, \bar{u}_{N}\right]$. The accuracy analysis consists in evaluating $p>0$ such that $H_{\alpha}(u)=$ $O\left(h^{p}\right)$ where

$$
H_{\alpha}(u)=\frac{1}{h_{\alpha}}\left[u\left(x_{\alpha+1 / 2}\right)-u\left(x_{\alpha-1 / 2}\right)\right]-\frac{1}{h_{\alpha}}\left[w_{\alpha}[U]\left(x_{\alpha+1 / 2}^{-}\right)-w_{\alpha}[U]\left(x_{\alpha-1 / 2}^{-}\right)\right] .
$$

Define the operator $L_{\alpha+1 / 2}^{-}(u)$ by

$$
L_{\alpha+1 / 2}^{-}(u)=u\left(x_{\alpha+1 / 2}\right)-w_{\alpha}[U]\left(x_{\alpha+1 / 2}^{-}\right) .
$$

Then $H_{\alpha}(u)$ becomes:

$$
H_{\alpha}(u)=\frac{1}{h_{\alpha}}\left[L_{\alpha+1 / 2}^{-}(u)-L_{\alpha-1 / 2}^{-}(u)\right] .
$$

In the particular case of a regular grid $\left(h_{\alpha}=h\right.$ for all $\alpha$ ), the operator $L_{\alpha+1 / 2}^{-}$is given by (see (4.15)):

$$
L_{\alpha+1 / 2}^{-}(u)=u\left(x_{\alpha+1 / 2}\right)-\left(\bar{u}_{\alpha}+\frac{1}{2} h \tilde{\sigma}_{\alpha}+\frac{1}{12} h^{2} \tilde{\theta}_{\alpha}+\frac{1}{48} h^{3} \tilde{\psi}_{\alpha}\right),
$$

where the notation is (see (4.12-4.13)):

$$
\left\{\begin{array}{l}
\bar{\sigma}_{\alpha}^{L S}=\frac{\bar{u}_{\alpha+1}-\bar{u}_{\alpha-1}}{2 h} \\
\tilde{\sigma}_{\alpha}=\frac{53}{48} \bar{\sigma}_{\alpha}^{L S}-\frac{5}{96} \bar{\sigma}_{\alpha+2}^{L S}-\frac{5}{96} \bar{\sigma}_{\alpha-2}^{L S}, \\
\tilde{\theta}_{\alpha}=\frac{1}{2 h}\left(\bar{\sigma}_{\alpha+1}^{L S}-\bar{\sigma}_{\alpha-1}^{L S}\right) \\
\tilde{\psi}_{\alpha}=\frac{1}{(2 h)^{2}}\left(\bar{\sigma}_{\alpha+2}^{L S}+\bar{\sigma}_{\alpha-2}^{L S}-2 \bar{\sigma}_{\alpha}^{L S}\right) .
\end{array}\right.
$$

The Taylor expansion of $H_{\alpha}(u)$ at $x_{\alpha}$ is (see (4.21)):

$$
H_{\alpha}(u)=-\frac{17}{384} h^{4} \partial_{x}^{(5)} u\left(x_{\alpha}\right)+\frac{229}{3840} h^{5} \partial_{x}^{(6)} u\left(x_{\alpha}\right)+O\left(h^{6}\right) .
$$

This proves Prop. 4.1, i.e. the fourth order accuracy of the scheme.

When $h_{\alpha}$ is non constant the evaluation of the order $p>$ such that $H_{\alpha}(u)=O\left(h^{p}\right)$ is more delicate. In particular, it cannot be performed by a Fourier symbol calculation. We show next how the Peano Kernel Theorem ([24], chap. 22, p.270) can provide a set of sufficient conditions on the irregularity of 
the grid in order for (9.2) to keep a high order accuracy. For simplicity of the presentation, the analysis is restricted to the case of the piecewise linear reconstruction defined by

$$
w_{\alpha}[V](x)=\left(v_{\alpha}+\sigma_{\alpha}[V]\left(x-x_{\alpha}\right)\right)
$$

where $\sigma_{\alpha}$ is the least squares slope $[12,15]$ given by:

$$
\sigma_{\alpha}=\frac{h_{\alpha, \alpha+1}}{h_{\alpha, \alpha-1}^{2}+h_{\alpha, \alpha+1}^{2}}\left(v_{\alpha+1}-v_{\alpha}\right)+\frac{h_{\alpha, \alpha-1}}{h_{\alpha, \alpha-1}^{2}+h_{\alpha, \alpha+1}^{2}}\left(v_{\alpha-1}-v_{\alpha}\right) .
$$

The same analysis can be extended to the cubic reconstruction case. Instead of (9.6) the operator $L_{\alpha+1 / 2}^{-}(u)$ is in the case of a linear reconstruction:

$$
L_{\alpha+1 / 2}^{-}(u)=u\left(x_{\alpha+1 / 2}\right)-\left(\bar{u}_{\alpha}+\frac{1}{2} h_{\alpha} \tilde{\sigma}_{\alpha}\right) .
$$

Since $L_{\alpha+1 / 2}^{-}(p)=0$ when $p(x)$ is any polynomial of degree 1 , the Peano Kernel Theorem implies that $L_{\alpha+1 / 2}^{-}$can be expressed as

$$
L_{\alpha+1 / 2}^{-}(u)=\int_{x_{\alpha-1 / 2}}^{x_{\alpha+1 / 2}} K_{\alpha+1 / 2}^{-}(\theta) u^{\prime \prime}(\theta) d \theta
$$

The kernel $K_{\alpha+1 / 2}^{-}(\theta)$ is

$$
K_{\alpha+1 / 2}^{-}(\theta)=\frac{1}{2} L_{\alpha+1 / 2, x}^{-}\left\{(x-\theta)_{+}\right\},
$$

where the operator $L_{\alpha+1 / 2}^{-}$is applied in the $x$-variable. A simple calculation shows that $K_{\alpha+1 / 2}^{-}(\theta)$ is expressed as the second order polynomial

$$
K_{\alpha+1 / 2}^{-}(\theta)=a_{\alpha}\left(x_{\alpha+1 / 2}-\theta\right)^{2}+b_{\alpha}\left(x_{\alpha+1 / 2}-\theta\right)+c_{\alpha},
$$

with coefficients (we denote $h_{\alpha+1 / 2}=\left(h_{\alpha}+h_{\alpha+1}\right) / 2$ ):

$$
\left\{\begin{aligned}
a_{\alpha} & =-\frac{1}{4 h_{\alpha}}+\frac{1}{8} \frac{h_{\alpha+1 / 2}-h_{\alpha-1 / 2}}{h_{\alpha+1 / 2}^{2}+h_{\alpha-1 / 2}^{2}} \\
b_{\alpha} & =\frac{1}{2}-\frac{1}{4} \frac{h_{\alpha} h_{\alpha+1 / 2}}{h_{\alpha+1 / 2}^{2}+h_{\alpha-1 / 2}^{2}} \\
c_{\alpha} & =-\frac{1}{8} \frac{h_{\alpha} h_{\alpha+1 / 2} h_{\alpha+1}}{h_{\alpha+1 / 2}^{2}+h_{\alpha-1 / 2}^{2}}
\end{aligned}\right.
$$

Consider now that $\alpha$ is a continuous parameter such that $\alpha \mapsto x(\alpha)$ describes the variation of the grid size. The grid size $h_{\alpha}=h(\alpha)$ is defined by

$$
h(\alpha)=x^{\prime}(\alpha) \text { with the additional assumption that } h(\alpha)=O(h) .
$$

The relations (9.15) become:

$$
\left\{\begin{array}{l}
a(\alpha)=-\frac{1}{4 h(\alpha)}+\frac{1}{8} \frac{h(\alpha+1 / 2)-h(\alpha-1 / 2)}{h^{2}(\alpha+1 / 2)+h^{2}(\alpha-1 / 2)} \\
b(\alpha)=\frac{1}{2}-\frac{1}{4} \frac{h(\alpha) h(\alpha+1 / 2)}{h^{2}(\alpha+1 / 2)+h^{2}(\alpha-1 / 2)} \\
c(\alpha)=-\frac{1}{8} \frac{h(\alpha) h(\alpha+1 / 2) h(\alpha+1)}{h^{2}(\alpha+1 / 2)+h^{2}(\alpha-1 / 2)}
\end{array}\right.
$$


Using a change of variable, $H_{\alpha}(u)$ in $(9.5)$ is expressed as:

$$
\begin{aligned}
H_{\alpha}(u)=\frac{1}{h(\alpha)} \int_{0}^{1} & \left\{\left[a(\alpha) h(\alpha)^{3} \tau^{2}+b(\alpha) h(\alpha)^{2} \tau+c(\alpha) h(\alpha)\right] u^{\prime \prime}(x(\alpha+1 / 2)-\tau h(\alpha))\right. \\
& \left.-\left[a(\alpha-1) h(\alpha-1)^{3} \tau^{2}+b(\alpha) h(\alpha-1)^{2} \tau+c(\alpha) h(\alpha-1)\right] u^{\prime \prime}(x(\alpha-1 / 2)-\tau h(\alpha-1))\right\} d \tau .
\end{aligned}
$$

Define $(\sigma, \tau) \in[\alpha-1, \alpha] \times[0,1] \mapsto A(\sigma, \tau)$ by

$$
A(\sigma, \tau)=a(\sigma) h(\sigma)^{3} \tau^{2}+b(\sigma) h(\sigma)^{2} \tau+c(\sigma) h(\sigma),
$$

where, for $\sigma \in[\alpha-1, \alpha]$, the values $a(\sigma), b(\sigma), c(\sigma)$ are given in (9.17) when replacing $h_{\alpha}$ by $h(\sigma)$ in the right-hand side. Then $H_{\alpha}(u)$ is rewritten as

$$
H_{\alpha}(u)=\frac{1}{h(\alpha)} \int_{[\alpha-1, \alpha] \times[0,1]} \frac{\partial}{\partial \sigma}\left[A(\sigma, \tau) u^{\prime \prime}(x(\sigma+1 / 2)-\tau h(\sigma))\right] d \sigma d \tau .
$$

Therefore by the mean value theorem for integrals, $\left|H_{\alpha}(u)\right|$ is estimated by

$$
\left|H_{\alpha}(u)\right| \leq \frac{1}{h(\alpha)} \int_{[\alpha-1, \alpha] \times[0,1]}\left|\frac{\partial}{\partial \sigma}\left[A(\sigma, \tau) u^{\prime \prime}(x(\sigma+1 / 2)-\tau h(\sigma))\right]\right| d \tau d \sigma .
$$

Finally we have

$$
\begin{aligned}
\frac{\partial}{\partial \sigma}\left[A(\sigma, \tau) u^{\prime \prime}(x(\sigma+1 / 2)-\tau h(\sigma))\right] & =\frac{\partial A(\sigma, \tau)}{\partial \sigma} u^{\prime \prime}(x(\sigma+1 / 2)-\tau h(\sigma)) \\
& +A(\sigma, \tau)\left(h(\sigma+1 / 2)-\tau h^{\prime}(\sigma)\right) u^{\prime \prime \prime}(x(\sigma+1 / 2)-\tau h(\sigma)) .
\end{aligned}
$$

This estimate leads to the following result:

Proposition 9.1. The scheme (9.2) is second order accurate withy respect to (9.1) under the sufficient condition on the variation of the grid $\alpha \mapsto x(\alpha)$, given by the relations

$$
\left\{\begin{array}{l}
\max _{\sigma, \tau}\left|\frac{\partial}{\partial \sigma} A(\sigma, \tau)\right| \leq C h^{3}, \\
\max _{\sigma, \tau}|A(\sigma, \tau)|\left(|h(\sigma)|+\left|h^{\prime}(\sigma)\right|\right) \leq C h^{3} .
\end{array}\right.
$$

Remark 9.2. In the case of a regular grid of size $h$, the function $h(\alpha)$ is the constant $h(\alpha)=h$. We have

$$
A(\sigma, \tau)=h^{2}\left(\frac{1}{4} \tau^{2}+\frac{3}{8} \tau-\frac{1}{16}\right) .
$$

Therefore $\partial_{\sigma} A=0$ and $A=O\left(h^{2}\right)$. Due to the fact that $h^{\prime}(\sigma)=0$, Prop 9.1 proves the second order accuracy of the scheme (9.2) and therefore we recover (9.8.)

Remark 9.3. Proposition 9.1 only claims that the accuracy is preserved when the grid is a slight perturbation of a regular grid in the sense of (9.23). The magnitude of the perturbation is measured by the variation of the function $A(\sigma, \tau)$. Therefore the problem of the accuracy analysis of the scheme (9.2) on grids with cells presenting large shape ratio remains an open question. 
Acknowledgement: The authors warmly thank F. Vuillot for his help and encouragement which was decisive for the final form of this paper. They also thank the referees for their sharp reading. Their suggestions greatly helped to enhance the presentation of this study.

[1] R. Abgrall and T. Sonar. On the use of Mühlbach expansions in the recovery step of ENO methods. Numerische Mathematik, 76:1-25, 1997.

[2] T. Barth. Numerical methods for conservation laws on structured and unstructured meshes. Technical report, VKI Lectures Series, 2003.

[3] T. Barth and M. Ohlberger. Finite Volume Methods: Foundation and Analysis. John Wiley \& Sons, 2004.

[4] T.J. Barth and P.O. Frederickson. Higher order solution of the euler equation on unstructured grids using quadratic reconstruction. In AIAA, number 90-0013 in AIAA, pages 1-12. AIAA, 1990.

[5] C. Bogey and C. Bailly. A family of low dispersive and low dissipative explicit schemes for flow noise and noise computations. J. Comput. Phys., 194:194-214, 2004.

[6] F. de la Puente, L. Sanders, and F. Vuillot. On LAGOON nose landing gear CFD/CAA computation over unstructured mesh using a ZDES approach. In Aviation,20th AIAA/CEAS Aeroacoustics Conference,20th AIAA/CEAS Aeroacoustics Conference, 16-20 June 2014, Atlanta, Ga, USA. AIAA. 2014-2763.

[7] M. Delanaye and J.A. Essers. Quadratic-reconstruction finite volume scheme for compressible flows on unstructured adaptive grids. AIAA Journal, 35(4):631-639, 1997.

[8] P. Deuflhard and A. Hohmann. Numerical Analysis in Modern Scientific Computing. An Introduction. Number 43 in TAM. Springer-Verlag, 2nd edition, 2003.

[9] M. Dumbser. Arbitrary high order $P^{N} P^{M}$ schemes on unstructured meshes for the compressible Navier-Stokes equations. Comput. Fluids, 39:60-76, 2010.

[10] G.H. Golub and C.F. Van Loan. Matrix Computations. John Hopkins University Press, 3-rd edition, 1996.

[11] W. Hackbusch. Tensor Spaces and Numerical Tensor Calculus, volume 42 of Springer Series in Computational Mathematics. Springer-Verlag, 2012.

[12] F. Haider. Discrétisation en maillage non structuré général - Applications à la LES (in french). PhD thesis, Univ. Paris 6 et Onera (the french aerospace lab), 2009.

[13] F. Haider, P. Brenner, B. Courbet, and J-P. Croisille. Efficient implementation of high order reconstruction in finite volume methods. In J. Fořt, J. Fürst, J. Halama, R. Herbin, and F. Hubert, editors, Finite Volumes for Complex Applications VI, Problems and Perspectives, pages 553-562. 6th FVCAA, Springer, 2011.

[14] F. Haider, P. Brenner, B. Courbet, and J-P. Croisille. Parallel implementation of $k$-exact finite volume reconstruction on unstructured grids. In R. Abgrall, H. Beaugendre, P.M. Congedo, C. Dobrzynski, V. Perrier, and M. Ricchiuto, editors, High Order Nonlinear numerical schemes for evolutionary PDEs, number 99 in Lect. Notes Comp. Sci. and Eng., pages 59-75. Springer, 2014.

[15] F. Haider, J-P. Croisille, and B. Courbet. Stability analysis of the cell centered finite-volume MUSCL method on unstructured grids. Numerische Mathematik, 113:555-600, 2009. 
[16] A. Harten. ENO Schemes with subcell resolution. J. Comput. Phys, 83:148-184, 1989.

[17] A. Harten and S. Chakravarthy. Multidimensional ENO schemes for general geometries. Technical Report 91-76, NASA-ICASE,Langley Research Center, Hampton., 1991.

[18] G. Jiang and C.W. Shu. Efficient implementation of weighted ENO schemes. J. Comput. Phys., 126:202-228, 1996.

[19] S. Khosla, P.J. Dionne, M.E. Lee, and C.E. Smith. Using fourth order spatial integration on unstructured meshes to reduce LES run time. In AIAA, number 2008-782 in 46th. AIAA, 2008.

[20] M. Lorteau, F. Cléro, and F. Vuillot. Recent progress in les computation for aeroacoustics of turbulent hot jet. Comparison to experiments and near field analysis. Number AIAA-2014-3057, Atlanta, Ga, USA, June 16-20 2014. AIAA/CEAS Aeroacoustics Conference.

[21] C. Michalak and C. Ollivier-Gooch. Unstructured high-order accurate finite-volume solutions of the Navier-Stokes equations. In AIAA, number 2009-954 in AIAA, pages 1-13, Orlando Florida, January 2009. AIAA.

[22] C. Ollivier-Gooch and M. Van Altena. A high-order accurate unstructured mesh finite-volume scheme for the advection-diffusion equation. J. Comput. Phys., 181(2):729-752, 2002.

[23] G. Pont, P. Brenner, P. Cinnella, B. Maugars, and J.-C. Robinet. Multiple-correction hybrid kexact schemes for high-order compressible RANS-LES simulations on fully unstructured grids. $J$. of Comput. Phys., 350:45 - 83, 2017.

[24] M.J.D. Powell. Approximation theory and methods. Cambridge University Press, 1981.

[25] G. Rahier, J. Prieur, F. Vuillot, N. Lupoglazoff, and A. Biancherin. Investigation of integral surface formulations for acoustic post-processing of unsteady aerodynamic jet simulations. Aerospace Science and Technology, 8(6):453 - 467, 2004.

[26] Yu.I. Shokin. The method of differential approximations. Springer Series in Computational Physics. Springer, 1983.

[27] C.-W. Shu. High order ENO and WENO schemes. In T.J. Barth and H. Deconinck, editors, High-Order Methods for Computational Physics, Lecture Notes in Computational Science and Engineering, pages 439-582. Springer-Verlag, Heidelberg, 1999.

[28] T. Sonar. On the construction of essentially non-oscillatory finite volume approximations to hyperbolic conservation laws on general triangulations: polynomial recovery, accuracy and stencil selection. Comput. methods Appl. mech. Engrg, 140:157-181, 1997.

[29] E. Toro. Riemann solvers and numerical methods for fluid dynamics. Springer, 3 edition, 2009.

[30] P.E. Vincent and A. Jameson. Facilitating the adoption of unstrutured hig-order methods amongst a wider community of fluid dynamicists. Math. Model. Nat. Phenom., 6(3):97-140, 2011.

[31] C-M. Winkler, J. Dorgan, and M. Mani. The effect of unstructured grid topology and resolution on simulations of decaying turbulence. 27th Int. Congress of the aeronautical sciences, 2010.

[32] W.J. Wiscombe and J.W Evans. Exponential-sum fitting of radiative transmission functions. Jour. Comput. Phys., 24:416-444, 1977. 
LaTeX Source Files
Click here to download LaTeX Source Files: paper-final-Sep-06-2018.tar.gz

LaTeX Source Files
Click here to download LaTeX Source Files: paper-final-Sep-06-2018.tar.gz

(

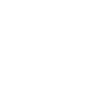

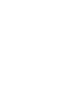

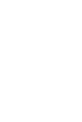

$\sqrt{10}$

. .

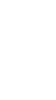

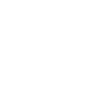

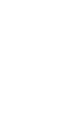
. .

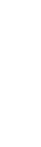

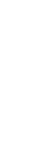
.

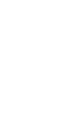

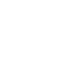

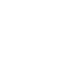

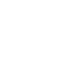

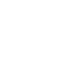

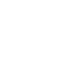

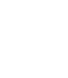

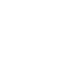

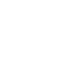

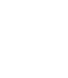

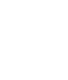

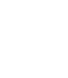

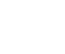

(1) 